\title{
On the Thomas-Fermi ground state in a harmonic potential
}

\author{
Clément Gallo ${ }^{\mathrm{a}, *}$ and Dmitry Pelinovsky ${ }^{\mathrm{b}, \uparrow}$ \\ a Institut de Mathématiques et de Modélisation de Montpellier, Université Montpellier II, \\ 34095 Montpellier, France \\ b Department of Mathematics, McMaster University, Hamilton, Ontario, Canada, L8S 4K1
}

October 6, 2010

\begin{abstract}
We study nonlinear ground states of the Gross-Pitaevskii equation in the space of one, two and three dimensions with a radially symmetric harmonic potential. The Thomas-Fermi approximation of ground states on various spatial scales was recently justified using variational methods. We justify here the Thomas-Fermi approximation on an uniform spatial scale using the Painlevé-II equation. In the space of one dimension, these results allow us to characterize the distribution of eigenvalues in the point spectrum of the Schrödinger operator associated with the nonlinear ground state.
\end{abstract}

\section{Introduction}

Recent experiments with Bose-Einstein condensates [PS] have stimulated new interest in the Gross-Pitaevskii equation with a harmonic potential. We take this equation in the form

$$
i u_{t}+\varepsilon^{2} \Delta u+\left(1-|x|^{2}\right) u-|u|^{2} u=0, \quad x \in \mathbb{R}^{d}, \quad t \in \mathbb{R}_{+},
$$

where the space dimension $d$ is one, two or three, $u(x, t) \in \mathbb{C}$ is the wave function of the repulsive Bose gas in the mean-field approximation, and $\varepsilon$ is a small parameter that corresponds to the Thomas-Fermi approximation of a nearly compact atomic cloud [Fer, T].

A ground state of the Bose-Einstein condensate is a positive, time-independent solution $u(x, t)=\eta_{\varepsilon}(x)$ of the Gross-Pitaevskii equation (1.1). More precisely, $\eta_{\varepsilon}: \mathbb{R}^{d} \mapsto \mathbb{R}$ satisfies the stationary Gross-Pitaevskii equation

$$
\varepsilon^{2} \Delta \eta_{\varepsilon}(x)+\left(1-|x|^{2}\right) \eta_{\varepsilon}(x)-\eta_{\varepsilon}^{3}(x)=0, \quad x \in \mathbb{R}^{d},
$$

$\eta_{\varepsilon}(x)>0$ for all $x \in \mathbb{R}^{d}$, and $\eta_{\varepsilon}$ has a finite energy $E_{\varepsilon}\left(\eta_{\varepsilon}\right)$, where $E_{\varepsilon}$ is given by

$$
E_{\varepsilon}(u)=\int_{\mathbb{R}^{d}}\left(\varepsilon^{2}|\nabla u|^{2}+\left(|x|^{2}-1\right) u^{2}+\frac{1}{2} u^{4}\right) d x .
$$

${ }^{*}$ C. Gallo was supported in part by the project ANR-07-BLAN-0250 of the Agence Nationale de la Recherche.

${ }^{\dagger}$ D. Pelinovsky was supported in part by the NSERC. 
For $d=2$, existence and uniqueness of a radially symmetric ground state $\eta_{\varepsilon}$ for a fixed, sufficiently small $\varepsilon>0$ is proven in Theorem 2.1 of Ignat \& Millot [IM] similarly to earlier works of Brezis \& Oswald [BO] and Aftalion, Alama, \& Bronsard [AAB] in bounded domains. It is also shown in $[\mathrm{IM}]$ that $\eta_{\varepsilon}(x)$ converges to $\eta_{0}(x)$ as $\varepsilon \rightarrow 0$ for all $x \in \mathbb{R}^{2}$, where $\eta_{0}$ is the Thomas-Fermi's compact function

$$
\eta_{0}(x)=\left\{\begin{array}{cl}
\left(1-|x|^{2}\right)^{1 / 2} & \text { for }|x|<1 \\
0 & \text { for }|x|>1 .
\end{array}\right.
$$

To be precise, Proposition 2.1 of [IM] states that for $d=2, \varepsilon>0$ sufficiently small,

$$
\begin{gathered}
0 \leqslant \eta_{\varepsilon}(x) \leqslant C \varepsilon^{1 / 3} \exp \left(\frac{1-|x|^{2}}{4 \varepsilon^{2 / 3}}\right) \text { for }|x| \geqslant 1, \\
0 \leqslant\left(1-|x|^{2}\right)^{1 / 2}-\eta_{\varepsilon}(x) \leqslant C \varepsilon^{1 / 3}\left(1-|x|^{2}\right)^{1 / 2} \text { for }|x| \leqslant 1-\varepsilon^{1 / 3},
\end{gathered}
$$

and

$$
\left\|\eta_{\varepsilon}-\eta_{0}\right\|_{C^{1}(K)} \leqslant C_{K} \varepsilon^{2}
$$

where $K$ is any compact subset of $\left\{x \in \mathbb{R}^{2}:|x|<1\right\}$ and $C$ and $C_{K}$ are $\varepsilon$-independent positive constants. The method used by Ignat \& Millot in the case $d=2$ to prove the existence of a radially symmetric ground state $\eta_{\varepsilon}$ can be extended to the cases $d=1,3$. The uniqueness of the positive, radially symmetric ground state does not follow from [IM] for $d=3$, but we can establish it by a different method, according to the following proposition. This proposition is proved in section 5 .

Proposition 1.1 The stationary Gross-Pitaevskii equation (1.2) has at most one positive radial solution in $L^{2}\left(\mathbb{R}^{d}\right)$, for any $d \geqslant 1$.

Our main goal in this paper is to prove a uniform asymptotic approximation of the ground state $\eta_{\varepsilon}$ on $\mathbb{R}^{d}$, in the limit $\varepsilon \rightarrow 0$, for $d=1,2,3$. At least two attempts have been made in physics literature [BTNN, KK] to establish connection between the nonlinear ground state $\eta_{\varepsilon}$ for $d=1$ and solutions of the Painlevé-II equation

$$
4 \nu^{\prime \prime}(y)+y \nu(y)-\nu^{3}(y)=0, \quad y \in \mathbb{R} .
$$

This equation arises as the formal limit as $\varepsilon \rightarrow 0$ of the differential equation satisfied by $\nu_{\varepsilon}$ :

$$
4\left(1-\varepsilon^{2 / 3} y\right) \nu_{\varepsilon}^{\prime \prime}(y)-2 \varepsilon^{2 / 3} d \nu_{\varepsilon}^{\prime}(y)+y \nu_{\varepsilon}(y)-\nu_{\varepsilon}^{3}(y)=0, \quad y \in\left(-\infty, \varepsilon^{-2 / 3}\right),
$$

where $\nu_{\varepsilon}$ is defined by

$$
\eta_{\varepsilon}(x)=\varepsilon^{1 / 3} \nu_{\varepsilon}(y), \quad y=\frac{1-|x|^{2}}{\varepsilon^{2 / 3}} .
$$

The convergence of $\eta_{\varepsilon}$ to $\eta_{0}$ as $\varepsilon \rightarrow 0$ suggests that we should consider the Hasting-McLeod solution $\nu_{0}$ of the Painlevé-II equation [HM], which is the unique solution of (1.7) such that

$$
\nu_{0}(y) \sim y^{1 / 2} \quad \text { as } \quad y \rightarrow+\infty \quad \text { and } \quad \nu_{0}(y) \rightarrow 0 \quad \text { as } \quad y \rightarrow-\infty .
$$


In both papers [BTNN, KK], the asymptotic solution $\eta_{\varepsilon}$ is constructed at three spatial scales

$$
\text { I : }|x| \leqslant 1-\varepsilon^{2 / 3}, \quad \text { II }:|x| \in\left(1-\varepsilon^{2 / 3}, 1+\varepsilon^{2 / 3}\right), \quad \text { and } \quad \text { III }:|x| \geqslant 1+\varepsilon^{2 / 3} .
$$

Solutions of the Painlevé-II equation (1.7) are used at the intermediate scale II for matching conditions and connection formulas between the WKB solutions at the inner scale I and the Airy function solutions at the outer scale III. The same formal approach is also developed in [ZAKP] for approximations of excited states of the stationary Gross-Pitaevskii equation in the case $d=1$.

We address the problem of uniform asymptotic approximations of the ground state $\eta_{\varepsilon}$ of the stationary Gross-Pitaevskii equation (1.2) using the Hasting-McLeod solution of the Painlevé-II equation (1.7). Our main result (Theorem 1) in Section 2 establishes this approximation on a rigorous level. In the case when $d=1$, we also study eigenvalues of the Schrödinger operator

$$
L_{+}^{\varepsilon}=-\varepsilon^{2} \partial_{x}^{2}+V_{\varepsilon}(x), \quad V_{\varepsilon}(x)=3 \eta_{\varepsilon}^{2}(x)-1+x^{2},
$$

that arises in the linearization of the stationary Gross-Pitaevskii equation (1.2) at the ground state $\eta_{\varepsilon}$. We prove in Section 3 that the spectrum of $L_{+}^{\varepsilon}$ in $L^{2}(\mathbb{R})$ consists of an infinite sequence of positive eigenvalues $\left\{\lambda_{n}^{\varepsilon}\right\}_{n \geqslant 1}$ such that for any fixed integer $k \geqslant 1$,

$$
\lambda_{2 k-1}^{\varepsilon}, \lambda_{2 k}^{\varepsilon} \sim \mu_{k} \varepsilon^{2 / 3} \quad \text { as } \varepsilon \rightarrow 0,
$$

where $\mu_{k}$ is the $k^{\text {th }}$ eigenvalue of the Schrödinger operator

$$
M_{0}=-4 \partial_{y}^{2}+W_{0}(y), \quad W_{0}(y)=3 \nu_{0}^{2}(y)-y .
$$

We note that $M_{0}$ arises in the linearization of the Painlevé-II equation (1.7) at the HastingMcLeod solution $\nu_{0}$. Therefore, the scaling transformation (1.8) leading to the Painlevé-II equation (1.7) becomes useful for analysis of eigenvalues of the Schrödinger operator $L_{+}^{\varepsilon}$.

It is clear from the shape of $\eta_{\varepsilon}$ that the operator $L_{+}^{\varepsilon}$ has a double-well potential $V_{\varepsilon}(x)$ with two symmetric minima converging to \pm 1 as $\varepsilon \rightarrow 0$, while the operator $M_{0}$ has a single-well potential $W_{0}(y)$. These facts explain both the asymptotic correspondence between eigenvalues of $L_{+}^{\varepsilon}$ and $M_{0}$ and the double degeneracy of each pair of eigenvalues in the asymptotic limit (1.9). Formal results of the semi-classical theory for the operator $L_{+}^{\varepsilon}$ are collected in Section 4.

While a different technique is exploited in our previous work [GP], the result (1.9) provides the same kind of asymptotic behaviour for the smallest eigenvalue of $L_{+}^{\varepsilon}$ as the one we obtained for the lowest eigenvalue of the simplified operator

$$
\tilde{L}_{+}^{\varepsilon}=-\varepsilon^{2} \partial_{x}^{2}+V_{0}(x), \quad V_{0}(x)=3 \eta_{0}^{2}(x)-1+x^{2} .
$$

The spectral stability of the ground state in the Gross-Pitaevskii equation (1.1) is deducted from the analysis of the symplectically coupled eigenvalue problem for Schrödinger operators $L_{+}^{\varepsilon}$ and $L_{-}^{\varepsilon}$, where

$$
L_{-}^{\varepsilon}=-\varepsilon^{2} \partial_{x}^{2}+\tilde{V}_{\varepsilon}(x), \quad \tilde{V}_{\varepsilon}(x)=\eta_{\varepsilon}^{2}(x)-1+x^{2}=\frac{\varepsilon^{2} \eta_{\varepsilon}^{\prime \prime}(x)}{\eta_{\varepsilon}(x)} .
$$

Unfortunately, the asymptotic scaling (1.8) leading to the Painlevé-II equation (1.7) does not give a correct scaling of the eigenvalues of $L_{-}^{\varepsilon}$ nor the eigenvalues of the spectral stability problem because the potential $\tilde{V}_{\varepsilon}(x)$ is a single well with a nearly flat bottom on the interval $[-1,1]$, which is mapped to $\left[0, \varepsilon^{-2 / 3}\right]$ by the change of variable $y=\left(1-x^{2}\right) / \varepsilon^{2 / 3}$. Analysis of the eigenvalues of the spectral stability problem and construction of excited states of the stationary Gross-Pitaevskii equation are two open problems beyond the scope of this article. 
Notations. If $A$ and $B$ are two quantities depending on a parameter $\varepsilon$ belonging to a neighborhood $\mathcal{E}$ of 0 ,

- $A(\varepsilon) \lesssim B(\varepsilon)$ indicates that there exists a positive constant $C$ such that

$$
A(\varepsilon) \leqslant C B(\varepsilon) \text { for every } \varepsilon \in \mathcal{E} \text {. }
$$

- $A(\varepsilon) \underset{\varepsilon \rightarrow 0}{\sim} B(\varepsilon)$ if $A(\varepsilon) / B(\varepsilon) \rightarrow 1$ as $\varepsilon \rightarrow 0$

- $A(\varepsilon)=\mathcal{O}(B(\varepsilon))$ as $\varepsilon \rightarrow 0$ if $A(\varepsilon) / B(\varepsilon)$ remains bounded as $\varepsilon \rightarrow 0$.

Let $F(x)$ be a function defined in a neighborhood of $\infty$. Given $\alpha \in \mathbb{R},\left\{f_{m}\right\}_{m \in \mathbb{N}} \in \mathbb{R}$, and $\gamma>0$, the notation

$$
F(x) \underset{x \rightarrow \infty}{\approx} x^{\alpha} \sum_{m=0}^{\infty} f_{m} x^{-\gamma m}
$$

means that for every $M \in \mathbb{N}$,

$$
F(x)-x^{\alpha} \sum_{m=0}^{M} f_{m} x^{-\gamma m}=\mathcal{O}\left(x^{\alpha-\gamma(M+1)}\right) \quad \text { as } x \rightarrow \infty,
$$

and, moreover, that the asymptotic series can be differentiated term by term.

We use the following spaces:

- $H^{\infty}(\mathbb{R})=\underset{s \geqslant 0}{\cap} H^{s}(\mathbb{R})$, where $H^{s}(\mathbb{R})$ is the standard Sobolev space.

- $L_{r}^{2}\left(\mathbb{R}^{d}\right)$ is the subspace of radially symmetric functions in $L^{2}\left(\mathbb{R}^{d}\right)$. Note that if $f(|\cdot|) \in$ $L_{r}^{2}\left(\mathbb{R}^{d}\right)$, then

$$
\|f(|\cdot|)\|_{L^{2}\left(\mathbb{R}^{d}\right)}=\left|\mathbb{S}^{d-1}\right| \int_{0}^{\infty} r^{d-1}|f(r)|^{2} d r
$$

where $\left|\mathbb{S}^{d-1}\right|$ is the surface of the unit sphere in $\mathbb{R}^{d}$. Similarly, $\left|\mathbb{B}^{d}\right|$ is the volume of the unit ball in $\mathbb{R}^{d}$.

\section{Uniform asymptotic expansion of $\eta_{\varepsilon}$}

In what follows, $d=1,2$ or 3 and $\varepsilon>0$ is sufficiently small such that, as it is proved in Theorem 2.1 of $[\mathrm{IM}]$, there exists a positive classical solution $\eta_{\varepsilon}$ of

$$
\varepsilon^{2} \Delta \eta_{\varepsilon}(x)+\left(1-|x|^{2}\right) \eta_{\varepsilon}(x)-\eta_{\varepsilon}^{3}(x)=0, \quad x \in \mathbb{R}^{d} .
$$

Moreover, this ground state $\eta_{\varepsilon}$ is radially symmetric, so that we can define a function $\nu_{\varepsilon}$ on $J_{\varepsilon}:=\left(-\infty, \varepsilon^{-2 / 3}\right]$ by

$$
\eta_{\varepsilon}(x)=\varepsilon^{1 / 3} \nu_{\varepsilon}\left(\frac{1-|x|^{2}}{\varepsilon^{2 / 3}}\right), \quad x \in \mathbb{R}^{d} .
$$


Let $y=\left(1-|x|^{2}\right) / \varepsilon^{2 / 3}$ be a new variable. Notice that $y$ covers once $J_{\varepsilon}$ as $|x|$ covers $\mathbb{R}_{+}$. It is equivalent for $\eta_{\varepsilon}$ to solve (2.1) and for $\nu_{\varepsilon}$ to solve the differential equation

$$
4\left(1-\varepsilon^{2 / 3} y\right) \nu_{\varepsilon}^{\prime \prime}(y)-2 \varepsilon^{2 / 3} d \nu_{\varepsilon}^{\prime}(y)+y \nu_{\varepsilon}(y)-\nu_{\varepsilon}^{3}(y)=0, \quad y \in J_{\varepsilon} .
$$

Let $N \geqslant 0$ be an integer. We look for $\nu_{\varepsilon}$ using the form

$$
\nu_{\varepsilon}(y)=\sum_{n=0}^{N} \varepsilon^{2 n / 3} \nu_{n}(y)+\varepsilon^{2(N+1) / 3} R_{N, \varepsilon}(y), \quad y \in J_{\varepsilon} .
$$

Expansion (2.4) provides a solution of equation (2.3) if $\left\{\nu_{n}\right\}_{0 \leqslant n \leqslant N}$ and $R_{N, \varepsilon}$ satisfy equations (2.5), (2.6) and (2.7) below.

- $\nu_{0}$ solves the Painlevé-II equation

$$
4 \nu_{0}^{\prime \prime}(y)+y \nu_{0}(y)-\nu_{0}^{3}(y)=0, \quad y \in \mathbb{R},
$$

- for $1 \leqslant n \leqslant N, \nu_{n}$ solves

$$
-4 \nu_{n}^{\prime \prime}(y)+W_{0}(y) \nu_{n}(y)=F_{n}(y), \quad y \in \mathbb{R},
$$

where

$$
W_{0}(y)=3 \nu_{0}^{2}(y)-y
$$

and

$$
F_{n}(y)=-\sum_{\substack{n_{1}, n_{2}, n_{3}<n \\ n_{1}+n_{2}+n_{3}=n}} \nu_{n_{1}}(y) \nu_{n_{2}}(y) \nu_{n_{3}}(y)-2 d \nu_{n-1}^{\prime}(y)-4 y \nu_{n-1}^{\prime \prime}(y)
$$

- $R_{N, \varepsilon}$ solves

$$
-4\left(1-\varepsilon^{2 / 3} y\right) R_{N, \varepsilon}^{\prime \prime}+2 \varepsilon^{2 / 3} d R_{N, \varepsilon}^{\prime}+W_{0} R_{N, \varepsilon}=F_{N, \varepsilon}\left(y, R_{N, \varepsilon}\right), \quad y \in J_{\varepsilon},
$$

where

$$
\begin{aligned}
& F_{N, \varepsilon}(y, R)=-\left(4 y \nu_{N}^{\prime \prime}+2 d \nu_{N}^{\prime}\right)-\sum_{n=0}^{2 N-1} \varepsilon^{2 n / 3} \sum_{\substack{n_{1}+n_{2}+n_{3}=n+N+1 \\
0 \leqslant n_{1}, n_{2}, n_{3} \leqslant N}} \nu_{n_{1}} \nu_{n_{2}} \nu_{n_{3}} \\
& -\left(3 \sum_{n=1}^{2 N} \varepsilon^{2 n / 3} \sum_{\substack{n_{1}+n_{2}=n \\
0 \leqslant n_{1}, n_{2} \leqslant N}} \nu_{n_{1}} \nu_{n_{2}}\right) R-\left(3 \sum_{n=N+1}^{2 N+1} \varepsilon^{2 n / 3} \nu_{n-(N+1)}\right) R^{2}-\varepsilon^{4(N+1) / 3} R^{3} .
\end{aligned}
$$

Notice that for $0 \leqslant n \leqslant N, \nu_{n}(y)$ is defined for all $y \in \mathbb{R}$ and does not depend on $\varepsilon$, whereas $R_{N, \varepsilon}(y)$ is a priori only defined for $y \in J_{\varepsilon}$.

Appropriate solutions of system (2.5), (2.6) and (2.7) enable us to prove the following theorem. 
Theorem 1 Let $\nu_{0}$ be the unique solution of the Painlevé II equation (2.5) such that

$$
\nu_{0}(y) \sim y^{1 / 2} \quad \text { as } y \rightarrow+\infty \quad \text { and } \quad \nu_{0}(y) \rightarrow 0 \quad \text { as } y \rightarrow-\infty .
$$

For $n \geqslant 1$, there exists a unique solution $\nu_{n}$ of equation (2.6) in $H^{\infty}(\mathbb{R})$. For every $N \geqslant 0$, there exists $\varepsilon_{N}>0$ and $C_{N}>0$ such that for every $0<\varepsilon<\varepsilon_{N}$, there is a solution $R_{N, \varepsilon} \in \mathcal{C}^{\infty} \cap L^{\infty}\left(J_{\varepsilon}\right)$ of equation (2.7) with

$$
\left\|R_{N, \varepsilon}\right\|_{L^{\infty}\left(J_{\varepsilon}\right)} \leqslant C_{N} \varepsilon^{-(d-1) / 3} \quad \text { and } \quad x \mapsto R_{N, \varepsilon}\left(\frac{1-|x|^{2}}{\varepsilon^{2 / 3}}\right) \in H^{2}\left(\mathbb{R}^{d}\right),
$$

such that the unique radially symmetric ground state of equation (2.1) in $L^{2}\left(\mathbb{R}^{d}\right)$ writes

$$
\eta_{\varepsilon}(x)=\varepsilon^{1 / 3} \sum_{n=0}^{N} \varepsilon^{2 n / 3} \nu_{n}\left(\frac{1-|x|^{2}}{\varepsilon^{2 / 3}}\right)+\varepsilon^{2 N / 3+1} R_{N, \varepsilon}\left(\frac{1-|x|^{2}}{\varepsilon^{2 / 3}}\right), \quad x \in \mathbb{R}^{d} .
$$

Remark 2.1 For $d=3$, the remainder term in (2.8) may have the same order as the last term in the sum, because of the growth of the upper bound on $\left\|R_{N, \varepsilon}\right\|_{L^{\infty}\left(J_{\varepsilon}\right)}$ as $\varepsilon \downarrow 0$.

Remark 2.2 The uniqueness of the ground state is proved in [IM] for $d=2$ (without assuming the radial symmetry of the ground state). The method of Ignat and Millot can be extended to the case $d=1$, but apparently not to $d=3$. Proposition 1.1 states the uniqueness of a positive, radially symmetric ground state in $L^{2}\left(\mathbb{R}^{d}\right)$ for $d=1,2,3$. This proposition is proved in Section 5.

The proof of Theorem 1 is described in the following three subsections. Notice first that it is sufficient to prove the Theorem for an arbitrarily large value of $N$. Indeed, for every integer $N_{0}>0$, the result of the Theorem for $N<N_{0}$ is a direct consequence of the result for $N=N_{0}$. Also, for convenience, we shall assume in the sequel that $N \geqslant 2$.

\subsection{Construction of $\nu_{n}$ for $0 \leqslant n \leqslant N$}

We are looking for a solution $\nu_{\varepsilon}(y)$ of equation (2.3) that satisfies the following limit as $\varepsilon \rightarrow 0$ :

$$
\varepsilon^{1 / 3} \nu_{\varepsilon}\left(\varepsilon^{-2 / 3}\left(1-x^{2}\right)\right) \underset{\varepsilon \rightarrow 0}{\longrightarrow}\left\{\begin{array}{cl}
\left(1-x^{2}\right)^{1 / 2} & \text { for } x \in[-1,1] \\
0 & \text { for }|x| \geqslant 1
\end{array}\right.
$$

Therefore, we choose $\nu_{0}(y)$ to be the unique solution of the Painlevé-II equation (2.5) that satisfies the asymptotic behavior $\nu_{0}(y) \sim y^{1 / 2}$ as $y \rightarrow+\infty$ and converges to zero as $y \rightarrow-\infty$. Existence and uniqueness of this solution are proved by Hastings \& McLeod [HM]. Asymptotic behaviour of $\nu_{0}(y)$ as $y \rightarrow \pm \infty$ is described in more details in Theorem 11.7 of [FIKN]. These results are combined together in the following proposition.

Proposition 2.1 [HM, FIKN] The Painlevé-II equation

$$
4 \nu^{\prime \prime}(y)+y \nu(y)-\nu^{3}(y)=0, \quad y \in \mathbb{R},
$$

admits a unique solution $\nu_{0} \in \mathcal{C}^{\infty}(\mathbb{R})$ such that

$$
\nu_{0}(y) \sim y^{1 / 2} \quad \text { as } \quad y \rightarrow+\infty \quad \text { and } \quad \nu_{0}(y) \rightarrow 0 \quad \text { as } \quad y \rightarrow-\infty .
$$


Moreover, $\nu_{0}$ is strictly increasing on $\mathbb{R}, \nu_{0}^{\prime \prime}$ has exactly one zero on $\mathbb{R}$, which is an inflection point of $\nu_{0}$. The behaviour of $\nu_{0}$ as $y \rightarrow-\infty$ is described by

$$
\nu_{0}(y)=\frac{1}{2 \sqrt{\pi}}(-2 y)^{-1 / 4} e^{-\frac{2}{3}(-2 y)^{3 / 2}}\left(1+\mathcal{O}\left(|y|^{-3 / 4}\right)\right) \underset{y \rightarrow-\infty}{\approx} 0,
$$

whereas as $y \rightarrow+\infty$, it is described by

$$
\nu_{0}(y) \underset{y \rightarrow+\infty}{\approx} y^{1 / 2} \sum_{n=0}^{\infty} \frac{b_{n}}{(2 y)^{3 n / 2}}
$$

where $b_{0}=1, b_{1}=0$, and for $n \geqslant 0$,

$$
b_{n+2}=4\left(9 n^{2}-1\right) b_{n}-\frac{1}{2} \sum_{m=1}^{n+1} b_{m} b_{n+2-m}-\frac{1}{2} \sum_{l=1}^{n+1} \sum_{m=1}^{n+2-l} b_{l} b_{m} b_{n+2-l-m} .
$$

Next, we construct $\nu_{n} \in H^{\infty}(\mathbb{R})$ for $n \geqslant 1$ by induction on $n$. For $n \geqslant 0$, we consider the following property:

$$
\left(\mathrm{H}_{\mathrm{n}}\right) \quad \forall k \in\{1, \ldots, n\}, \quad\left\{\begin{array}{l}
\bullet \nu_{k} \in H^{\infty}(\mathbb{R}) \text { solves }(2.6)(\text { with } n \text { replaced by } k), \\
\bullet \nu_{k}(y) \underset{y \rightarrow+\infty}{\approx} y^{\beta-2 k} \sum_{m=0}^{\infty} g_{k, m} y^{-3 m / 2} \text { for some }\left\{g_{k, m}\right\}_{m \in \mathbb{N}}, \\
\bullet \nu_{k}(y) \underset{y \rightarrow-\infty}{\approx} 0,
\end{array}\right.
$$

where

$$
\beta= \begin{cases}-5 / 2 & \text { if } d=1, \\ 1 / 2 & \text { if } d=2,3 .\end{cases}
$$

$\left(\mathrm{H}_{0}\right)$ is empty and, therefore, true by convention. Fix $n \geqslant 1$ and assume that $\left(\mathrm{H}_{\mathrm{n}-1}\right)$ is true. We are going to construct $\nu_{n}$ such that $\left(\mathrm{H}_{\mathrm{n}}\right)$ is satisfied. We will make use of the following two lemmas, which are proved in Sections 6 and 7.

Lemma 2.1 Let $W \in \mathcal{C}^{1}(\mathbb{R})$ such that $W^{\prime} \in L^{\infty}\left(\mathbb{R}_{+}\right)$and there exists $C_{0}, C_{+}, A_{+}>0$ with

$$
W(x) \geqslant C_{+} x \quad \text { for } \quad x \geqslant A_{+}, \quad W(x) \geqslant C_{0} \quad \text { for } \quad x \in \mathbb{R}, \quad \text { and } \quad W^{\prime}(x) \geqslant 0 \text { for } \quad x \geqslant A_{+} .
$$

Let $f \in L^{2}(\mathbb{R})$ such that $x^{\alpha} f \in L^{\infty}\left(A_{+},+\infty\right)$ for some $\alpha>0$. Let

$$
\varphi=\left(-\partial_{x}^{2}+W\right)^{-1} f \in H^{1}(\mathbb{R}) .
$$

Then, as $x \rightarrow+\infty$,

$$
\varphi(x)=\mathcal{O}\left(x^{-(\alpha+1)}\right) .
$$

Moreover, if $f$ and $W$ admit asymptotic series

$$
f(x) \underset{x \rightarrow+\infty}{\approx} x^{-\alpha} \sum_{m=0}^{+\infty} c_{m} x^{-\gamma m}, \quad W(x) \underset{x \rightarrow+\infty}{\approx} x \sum_{m=0}^{+\infty} v_{m} x^{-\gamma m}
$$


for some coefficients $\left\{c_{m}\right\}_{m \in \mathbb{N}},\left\{v_{m}\right\}_{m \in \mathbb{N}}$ and $\gamma>0$ such that $3 / \gamma$ is an integer, then $\varphi$ admits an asymptotic series

$$
\varphi(x) \underset{x \rightarrow+\infty}{\approx} x^{-(\alpha+1)} \sum_{m=0}^{+\infty} d_{m} x^{-\gamma m}
$$

for some coefficients $\left\{d_{m}\right\}_{m \in \mathbb{N}}$. In particular, as $x \rightarrow+\infty$,

$$
\varphi^{\prime}(x)=\mathcal{O}\left(x^{-(\alpha+2)}\right), \quad \varphi^{\prime \prime}(x)=\mathcal{O}\left(x^{-(\alpha+3)}\right) .
$$

Lemma 2.2 Let $W_{0}(y):=3 \nu_{0}^{2}(y)-y$, where $\nu_{0}(y)$ is the solution of the Painlevé-II equation (2.5) given in Proposition 2.1. Then,

$$
W_{\min }:=\inf _{y \in \mathbb{R}} W_{0}(y)>0 .
$$

¿From the asymptotic behaviors of $\nu_{0}(y)$ as $y \rightarrow \pm \infty$, we infer that

$$
W_{0}(y) \sim 2 y \quad \text { as } \quad y \rightarrow+\infty \text { and } W_{0}(y) \sim-y \quad \text { as } \quad y \rightarrow-\infty .
$$

Let us consider the operator

$$
M_{0}:=-4 \partial_{y}^{2}+W_{0}(y)
$$

on $L^{2}(\mathbb{R})$ with the domain,

$$
\operatorname{Dom}\left(M_{0}\right)=\left\{u \in L^{2}(\mathbb{R}): \quad-4 u^{\prime \prime}+W_{0} u \in L^{2}(\mathbb{R})\right\} .
$$

The Schrödinger operator $M_{0}$ arises in the linearization of the Painlevé-II equation at $\nu=\nu_{0}$. The spectrum of $M_{0}$ is purely discrete and, thanks to Lemma 2.2, it consists of a sequence of strictly positive eigenvalues which goes to infinity. If $n=1$, it follows from the choice of $\nu_{0}$ and from properties (2.9)-(2.10) that

$$
F_{1}(y) \underset{y \rightarrow+\infty}{\approx}(1-d) y^{-1 / 2}+y^{-7 / 2} \sum_{m=0}^{+\infty} 3(m+2) b_{m+2} 2^{-3(m+2) / 2}(1-3(m+2)) y^{-3 m / 2}
$$

and

$$
F_{1}(y) \underset{y \rightarrow-\infty}{\approx} 0 .
$$

Thanks to Lemma 2.2, we can look for $\nu_{1}$ solution of (2.6) with $n=1$ in the form

$$
\nu_{1}(y)=\frac{(1-d) \Phi(y)}{W_{0}(y) y^{1 / 2}}+\tilde{\nu}_{1}(y), \quad y \in \mathbb{R}
$$

where $\Phi \in \mathcal{C}^{\infty}(\mathbb{R})$ is such that $\Phi(y) \equiv 0$ if $y \leqslant 1 / 2, \Phi(y) \equiv 1$ if $y \geqslant 1$. Then, $\tilde{\nu}_{1}$ has to solve

$$
-4 \tilde{\nu}_{1}^{\prime \prime}(y)+W_{0}(y) \tilde{\nu}_{1}(y)=\tilde{F}_{1}(y):=F_{1}(y)-(1-d) y^{-1 / 2} \Phi(y)+4 \frac{d^{2}}{d y^{2}}\left(\frac{(1-d) \Phi(y)}{W_{0}(y) y^{1 / 2}}\right), y \in \mathbb{R} .
$$

¿From the asymptotic expansions (2.9)-(2.10) of $\nu_{0}$, we infer that $W_{0}$ also admits asymptotic expansions as $y \rightarrow \pm \infty$. Since $\nu_{0} \in \mathcal{C}^{\infty}(\mathbb{R})$, it follows from (2.15), (2.16), (2.17) and (2.18) 
that $\tilde{F}_{1} \in H^{\infty}(\mathbb{R})$. Then, property $\left(\mathrm{H}_{1}\right)$ follows from Lemma 2.1 applied on the one side to $\tilde{\nu}_{1}:=M_{0}^{-1} \tilde{F}_{1}$ with $\alpha=7 / 2$, so that $\tilde{\nu}_{1}(y)=\mathcal{O}\left(y^{-9 / 2}\right)$ as $y \rightarrow+\infty$, and on the other side to $y \mapsto \tilde{\nu}_{1}(-y)$ with $\alpha$ arbitrarily large. Furthermore, if $n \geqslant 2$, we have

$$
F_{n}(y)=-\sum_{\substack{0<n_{1}, n_{2}, n_{3}<n \\ n_{1}+n_{2}+n_{3}=n}} \nu_{n_{1}}(y) \nu_{n_{2}}(y) \nu_{n_{3}}(y)-3 \sum_{\substack{0<n_{1}, n_{2}<n \\ n_{1}+n_{2}=n}} \nu_{0}(y) \nu_{n_{1}}(y) \nu_{n_{2}}(y)-2 d \nu_{n-1}^{\prime}(y)-4 y \nu_{n-1}^{\prime \prime}(y) .
$$

Thanks to $\left(\mathrm{H}_{\mathrm{n}-1}\right)$, all the terms in the right hand side admit an asymptotic expansion at $\pm \infty$. More precisely,

$$
F_{n}(y) \underset{y \rightarrow+\infty}{\approx} y^{\beta+1-2 n} \sum_{m=0}^{+\infty} f_{n, m} y^{-3 m / 2}
$$

for some coefficients $\left\{f_{n, m}\right\}_{m \in \mathbb{N}}$, whereas

$$
F_{n}(y) \underset{y \rightarrow-\infty}{\approx} 0 .
$$

Since $F_{n} \in \mathcal{C}^{\infty}(\mathbb{R})$ and $n \geqslant 2$, we deduce that $F_{n} \in H^{\infty}(\mathbb{R})$, and we can define $\nu_{n}=M_{0}^{-1} F_{n} \in$ $H^{\infty}(\mathbb{R})$. By Lemma 2.1 with $\gamma=3 / 2$ and $\alpha=2 n-\beta-1$, we then have

$$
\nu_{n}(y) \underset{y \rightarrow+\infty}{\approx} y^{\beta-2 n} \sum_{m=0}^{+\infty} g_{n, m} y^{-3 m / 2}
$$

for some coefficients $\left\{g_{n, m}\right\}_{m \in \mathbb{N}}$, and

$$
\nu_{n}(y) \underset{y \rightarrow-\infty}{\approx} 0
$$

where we have applied Lemma 2.1 with $\gamma=3 / 2$ to the function $\nu_{n}(-y)$. Therefore, $\left(\mathrm{H}_{\mathrm{n}}\right)$ is true, which completes the construction by induction of the sequence of solutions $\left\{\nu_{n}(y)\right\}_{n \geqslant 1}$ of the inhomogeneous equations (2.6).

\subsection{Construction of $R_{N, \varepsilon}$}

In this subsection, we construct a solution $R_{N, \varepsilon}$ to equation (2.7), such that given the $\nu_{n}$ 's constructed in subsection 2.1, expansion (2.4) provides a solution of equation (2.3). The solution $R_{N, \varepsilon}$ of equation (2.7) is obtained by a fixed point argument. In order to explain the functional framework in which the fixed point theorem will be applied, let us first introduce the functional spaces

$$
L_{\varepsilon}^{2}=\left\{u \in L_{\mathrm{loc}}^{1}\left(J_{\varepsilon}\right): \quad\left(1-\varepsilon^{2 / 3} y\right)^{d / 4-1 / 2} u \in L^{2}\left(J_{\varepsilon}\right)\right\}
$$

and

$$
H_{\varepsilon}^{1}=\left\{u \in L_{\varepsilon}^{2}: \quad\left(1-\varepsilon^{2 / 3} y\right)^{d / 4} u^{\prime} \in L^{2}\left(J_{\varepsilon}\right) \text { and }\left(1-\varepsilon^{2 / 3} y\right)^{d / 4-1 / 2} W_{0}^{1 / 2} u \in L^{2}\left(J_{\varepsilon}\right)\right\},
$$

endowed with their respective squared norms

$$
\|u\|_{\varepsilon}^{2}:=\|u\|_{L_{\varepsilon}^{2}}^{2}=\int_{-\infty}^{\varepsilon^{-2 / 3}}\left(1-\varepsilon^{2 / 3} y\right)^{d / 2-1} u^{2} d y
$$


and

$$
\|u\|_{H_{\varepsilon}^{1}}^{2}:=\int_{-\infty}^{\varepsilon^{-2 / 3}}\left[4\left(1-\varepsilon^{2 / 3} y\right)^{d / 2}\left|u^{\prime}\right|^{2}+\left(1-\varepsilon^{2 / 3} y\right)^{d / 2-1} W_{0} u^{2}\right] d y .
$$

We are looking for a solution $R_{N, \varepsilon}(y)$ of Equation (2.7) on $J_{\varepsilon}$ such that the function $R_{N, \varepsilon}\left(\varepsilon^{-2 / 3}(1-\right.$ $\left.|x|^{2}\right)$ ) is regular on $\mathbb{R}^{d}$. As a result, it is convenient for the sequel to introduce the map $T^{\varepsilon}: L_{\varepsilon}^{2} \mapsto$ $L_{r}^{2}\left(\mathbb{R}^{d}\right)$ defined for $u \in L_{\varepsilon}^{2}$ by

$$
\left(T^{\varepsilon} u\right)(z):=u\left(\varepsilon^{-2 / 3}-\varepsilon^{2 / 3}|z|^{2}\right),
$$

which makes the link between functions defined on $J_{\varepsilon}$ and radial functions defined on $\mathbb{R}^{d}$, in terms of the variable $z=\varepsilon^{-2 / 3} x \in \mathbb{R}^{d}$. An easy calculation shows that $T^{\varepsilon}$ is a bijection from $L_{\varepsilon}^{2}$ into $L_{r}^{2}\left(\mathbb{R}^{d}\right)$, and that for every $u \in L_{\varepsilon}^{2}$,

$$
\left\|T^{\varepsilon} u\right\|_{L^{2}\left(\mathbb{R}^{d}\right)}^{2}=\frac{\left|\mathbb{S}^{d-1}\right|}{2 \varepsilon^{2(d-1) / 3}}\|u\|_{\varepsilon}^{2}
$$

Moreover, $T^{\varepsilon}$ induces a bijection from $H_{\varepsilon}^{1}$ into

$$
Q_{\varepsilon}:=\left\{u \in L^{2}\left(\mathbb{R}^{d}\right): \int_{\mathbb{R}^{d}}\left[|\nabla u|^{2}+W_{0}\left(\varepsilon^{-2 / 3}-\varepsilon^{2 / 3}|z|^{2}\right)|u|^{2}\right] d z<\infty\right\}
$$

and for every $u \in H_{\varepsilon}^{1}$,

$$
\left\|T^{\varepsilon} u\right\|_{Q_{\varepsilon}}^{2}=\int_{\mathbb{R}^{d}}\left[\left|\nabla T^{\varepsilon} u\right|^{2}+W_{0}\left(\varepsilon^{-2 / 3}-\varepsilon^{2 / 3}|z|^{2}\right)\left|T^{\varepsilon} u\right|^{2}\right] d z=\frac{\left|\mathbb{S}^{d-1}\right|}{2 \varepsilon^{2(d-1) / 3}}\|u\|_{H_{\varepsilon}^{1}}^{2} .
$$

Let us rewrite equation (2.7) for the remainder term $R_{N, \varepsilon}(y)$ in the operator form

$$
M^{\varepsilon} R_{N, \varepsilon}(y)=F_{N, \varepsilon}\left(y, R_{N, \varepsilon}\right), \quad y \in J_{\varepsilon},
$$

where $M^{\varepsilon}$ is the self-adjoint operator on $L_{\varepsilon}^{2}$ defined by

$$
\left\{\begin{array}{l}
M^{\varepsilon}:=-4\left(1-\varepsilon^{2 / 3} y\right)^{-d / 2+1} \partial_{y}\left(1-\varepsilon^{2 / 3} y\right)^{d / 2} \partial_{y}+W_{0}(y)=\left(T^{\varepsilon}\right)^{-1} K^{\varepsilon} T^{\varepsilon} \\
\operatorname{Dom}\left(M^{\varepsilon}\right)=\left\{u \in L_{\varepsilon}^{2}: K^{\varepsilon} T_{\varepsilon} u \in L^{2}\left(\mathbb{R}^{d}\right)\right\}
\end{array}\right.
$$

and $K^{\varepsilon}$ denotes the Schrödinger operator on $L^{2}\left(\mathbb{R}^{d}\right)$,

$$
K^{\varepsilon}:=-\Delta+W_{0}\left(\varepsilon^{-2 / 3}-\varepsilon^{2 / 3}|z|^{2}\right) .
$$

The solution $R_{N, \varepsilon}$ of the nonlinear equation (2.21) will be obtained from the fixed point theorem applied to the map

$$
\Phi_{N, \varepsilon}: R \mapsto\left(M^{\varepsilon}\right)^{-1} F_{N, \varepsilon}(\cdot, R),
$$

which will be shown to be continuous from $H_{\varepsilon}^{1}$ into itself. First, we shall prove the following lemma.

Lemma 2.3 The operator $M^{\varepsilon}$ is invertible, and for every $f \in L_{\varepsilon}^{2}$,

$$
\left\|\left(M^{\varepsilon}\right)^{-1} f\right\|_{H_{\varepsilon}^{1}} \leqslant W_{\min }^{-1 / 2}\|f\|_{\varepsilon} .
$$


Proof. Let us consider the continuous, bilinear, coercive form on $Q_{\varepsilon}$ defined by

$$
a(u, v)=\int_{\mathbb{R}^{d}}\left[\nabla u \nabla v+W_{0}\left(\varepsilon^{-2 / 3}-\varepsilon^{2 / 3}|z|^{2}\right) u v\right] d z .
$$

By the Cauchy-Schwarz inequality and Lemma 2.2, for every $f \in L_{\varepsilon}^{2}$,

$$
v \mapsto \int_{\mathbb{R}^{d}} T^{\varepsilon} f v d z
$$

defines a continuous linear form on $Q_{\varepsilon}$. Thus, by the Lax-Milgram Theorem [GT], there exists a unique $\psi \in Q_{\varepsilon}$ such that for every $v \in Q_{\varepsilon}$,

$$
a(\psi, v)=\int_{\mathbb{R}^{d}} T^{\varepsilon} f v d z
$$

Moreover, $\psi \in Q_{\varepsilon}$ is radial and satisfies

$$
K^{\varepsilon} \psi=T^{\varepsilon} f \quad \text { in } \quad \mathcal{D}^{\prime}\left(\mathbb{R}^{d}\right) .
$$

Thus, $\varphi:=\left(T^{\varepsilon}\right)^{-1} \psi \in H_{\varepsilon}^{1} \cap \operatorname{Dom}\left(M^{\varepsilon}\right)$ satisfies

$$
M^{\varepsilon} \varphi=f .
$$

¿From (2.20) and a calculation similar to (2.19), we also check that

$$
\begin{aligned}
\|\varphi\|_{H_{\varepsilon}^{1}}^{2} & =\frac{2 \varepsilon^{2(d-1) / 3}}{\left|\mathbb{S}^{d-1}\right|} a(\psi, \psi)=\frac{2 \varepsilon^{2(d-1) / 3}}{\left|\mathbb{S}^{d-1}\right|} \int_{\mathbb{R}^{d}} T^{\varepsilon} f \psi d z=\int_{-\infty}^{\varepsilon^{-2 / 3}}\left(1-\varepsilon^{2 / 3} y\right)^{d / 2-1} f \varphi d y \\
& \leqslant\|f\|_{\varepsilon}\|\varphi\|_{\varepsilon} \leqslant W_{\min }^{-1 / 2}\|f\|_{\varepsilon}\|\varphi\|_{H_{\varepsilon}^{1}}
\end{aligned}
$$

from which the upper bound on $\varphi=\left(M^{\varepsilon}\right)^{-1} f$ in $H_{\varepsilon}^{1}$ follows.

Next, we prove that $R \mapsto F_{N, \varepsilon}(\cdot, R)$ continuously maps $H_{\varepsilon}^{1}$ into $L_{\varepsilon}^{2}$. We write

$$
F_{N, \varepsilon}(y, R)=F_{N, 0}(y)+G_{N, \varepsilon}(y, R),
$$

where

$$
F_{N, 0}=-\left(4 y \nu_{N}^{\prime \prime}+2 d \nu_{N}^{\prime}\right)-\sum_{\substack{n_{1}+n_{2}+n_{3}=N+1 \\ 0 \leqslant n_{1}, n_{2}, n_{3} \leqslant N}} \nu_{n_{1}} \nu_{n_{2}} \nu_{n_{3}}
$$

and

$$
\begin{aligned}
G_{N, \varepsilon}= & -\sum_{n=1}^{2 N-1} \varepsilon^{2 n / 3} \sum_{\substack{n_{1}+n_{2}+n_{3}=n+N+1 \\
0 \leqslant n_{1}, n_{2}, n_{3} \leqslant N}} \nu_{n_{1}} \nu_{n_{2}} \nu_{n_{3}}-\left(3 \sum_{n=1}^{2 N} \varepsilon^{2 n / 3} \sum_{\substack{n_{1}+n_{2}=n \\
0 \leqslant n_{1}, n_{2} \leqslant N}} \nu_{n_{1}} \nu_{n_{2}}\right) R \\
& -\left(3 \sum_{n=N+1}^{2 N+1} \varepsilon^{2 n / 3} \nu_{n-(N+1)}\right) R^{2}-\varepsilon^{4(N+1) / 3} R^{3}
\end{aligned}
$$


We first show that $F_{N, 0} \in L_{\varepsilon}^{2}$. Indeed, from the properties of the $\nu_{n}$ 's, we infer that

$$
F_{N, 0}(y) \underset{y \rightarrow-\infty}{\approx} 0
$$

and $F_{N, 0}$ also admits an asymptotic expansion as $y \rightarrow+\infty$, with

$$
4 y \nu_{N}^{\prime \prime}(y)+2 d \nu_{N}^{\prime}(y)=\mathcal{O}\left(y^{\beta-1-2 N}\right)
$$

and if $n_{1}+n_{2}+n_{3}=N+1$,

$$
\nu_{n_{1}} \nu_{n_{2}} \nu_{n_{3}}(y)= \begin{cases}\mathcal{O}\left(y^{-19 / 2-2 N}\right) & \text { if } d=1 \text { and } n_{1}, n_{2}, n_{3}>0 \\ \mathcal{O}\left(y^{-13 / 2-2 N}\right) & \text { if } d=1 \text { and } n_{1} \text { or } n_{2} \text { or } n_{3}=0 \\ \mathcal{O}\left(y^{-1 / 2-2 N}\right) & \text { if } d \geqslant 2\end{cases}
$$

(notice that $n_{1}+n_{2}+n_{3}=N+1$ with $0 \leqslant n_{1}, n_{2}, n_{3} \leqslant N$ implies that at most one of the numbers $n_{1}, n_{2}, n_{3}$ is equal to 0 ). Since $N \geqslant 2$, we deduce that in any case,

$$
F_{N, 0}(y)=\mathcal{O}\left(y^{-9 / 2}\right) \text { as } y \rightarrow+\infty, \text { while } \quad F_{N, 0}(y) \underset{y \rightarrow-\infty}{\approx} 0 .
$$

Therefore, for $\alpha>0$ sufficiently large and $\varepsilon<1$,

$$
\int_{-\infty}^{\varepsilon^{-2 / 3}}\left(1-\varepsilon^{2 / 3} y\right)^{d / 2-1} F_{N, 0}^{2} d y \lesssim \int_{-\infty}^{1}(1+|y|)^{-2 \alpha} d y+\int_{1}^{\varepsilon^{-2 / 3}} y^{-9}\left(1-\varepsilon^{2 / 3} y\right)^{d / 2-1} d y .
$$

In the case $d=1$, the second integral in the right hand side is estimated by

$$
\begin{aligned}
\int_{1}^{\varepsilon^{-2 / 3}} y^{-9}\left(1-\varepsilon^{2 / 3} y\right)^{-1 / 2} d y & \lesssim \varepsilon^{16 / 3} \int_{\varepsilon^{2 / 3}}^{1} \frac{z^{-9}}{(1-z)^{1 / 2}} d z \\
& \lesssim \varepsilon^{16 / 3} \sqrt{2} \int_{\varepsilon^{2 / 3}}^{1 / 2} z^{-9} d z+\frac{\varepsilon^{16 / 3}}{2^{9}} \int_{1 / 2}^{1} \frac{1}{(1-z)^{1 / 2}} d z \lesssim 1
\end{aligned}
$$

whereas for $d \geqslant 2$,

$$
\int_{1}^{\varepsilon^{-2 / 3}} y^{-9}\left(1-\varepsilon^{2 / 3} y\right)^{d / 2-1} d y \lesssim \int_{1}^{\varepsilon^{-2 / 3}} y^{-9} d y \lesssim 1
$$

Therefore in both cases $F_{N, 0} \in L_{\varepsilon}^{2}$ and

$$
\left\|F_{N, 0}\right\|_{\varepsilon} \lesssim 1
$$

Similarly, the term which does not depend on $R$ in the right hand side of $(2.24)$ is $\mathcal{O}_{L_{\varepsilon}^{2}}\left(\varepsilon^{2 / 3}\right)$. Let $R \in H_{\varepsilon}^{1}$. To estimate the linear term in $R$ in the definition of $G_{N, \varepsilon}$, notice that if $n_{1}+n_{2}=$ $n \geqslant 1$, then $n_{1}$ or $n_{2}$ is not equal to 0 , thus $\nu_{n_{1}} \nu_{n_{2}}(y)=\mathcal{O}\left(y^{-1}\right)$ as $y \rightarrow+\infty$. In particular, $\nu_{n_{1}} \nu_{n_{2}} \in L^{\infty}(\mathbb{R})$ and

$$
\left\|\nu_{n_{1}} \nu_{n_{2}} R\right\|_{\varepsilon} \leqslant\left\|\nu_{n_{1}} \nu_{n_{2}}\right\|_{L^{\infty}(\mathbb{R})}\|R\|_{\varepsilon} \leqslant\left\|\nu_{n_{1}} \nu_{n_{2}}\right\|_{L^{\infty}(\mathbb{R})} W_{\min }^{-1 / 2}\|R\|_{H_{\varepsilon}^{1}} \lesssim\|R\|_{H_{\varepsilon}^{1}} .
$$

In order to estimate the quadratic and cubic terms in the right hand side of (2.24), the following lemma will be useful. 
Lemma 2.4 Let $p=1,2$ or 3 . There exists a $\varepsilon$-independent constant $C>0$ such that for every $\varepsilon>0$, if $u \in H_{\varepsilon}^{1}$ then $u^{p} \in L_{\varepsilon}^{2}$ and

$$
\left\|u^{p}\right\|_{\varepsilon} \leqslant C \varepsilon^{-(p-1)(d-1) / 3}\|u\|_{H_{\varepsilon}^{1}}^{p} .
$$

Proof. Let $u \in H_{\varepsilon}^{1}$. We have checked in $(2.20)$ that $T^{\varepsilon} u \in Q_{\varepsilon} \subset H^{1}\left(\mathbb{R}^{d}\right)$ and

$$
\left\|T^{\varepsilon} u\right\|_{H^{1}\left(\mathbb{R}^{d}\right)} \lesssim\left\|T^{\varepsilon} u\right\|_{Q_{\varepsilon}} \lesssim \varepsilon^{-(d-1) / 3}\|u\|_{H_{\varepsilon}^{1}} .
$$

By Sobolev embeddings, it follows that $T^{\varepsilon} u \in L^{2 p}\left(\mathbb{R}^{d}\right)$, and

$$
\begin{aligned}
\left\|u^{p}\right\|_{\varepsilon} & \lesssim \varepsilon^{(d-1) / 3}\left\|T^{\varepsilon}\left(u^{p}\right)\right\|_{L^{2}\left(\mathbb{R}^{d}\right)}=\varepsilon^{(d-1) / 3}\left\|T^{\varepsilon} u\right\|_{L^{2 p}\left(\mathbb{R}^{d}\right)}^{p} \\
& \lesssim \varepsilon^{(d-1) / 3}\left\|T^{\varepsilon} u\right\|_{H^{1}\left(\mathbb{R}^{d}\right)}^{p} \lesssim \varepsilon^{-(p-1)(d-1) / 3}\|u\|_{H_{\varepsilon}^{1}}^{p}
\end{aligned}
$$

where we have also made use of (2.19) with $u$ replaced by $u^{p}$.

Remark 2.3 The statement of Lemma 2.4 can be extended for all values of $p$ for which $H^{1}\left(\mathbb{R}^{d}\right)$ is continuously embedded into $L^{2 p}\left(\mathbb{R}^{d}\right)$, that is $1 \leqslant p \leqslant \infty$ for $d=1,1 \leqslant p<\infty$ for $d=2$ and $1 \leqslant p \leqslant 3$ for $d=3$.

Thanks to Lemma 2.4, for any integer $k \geqslant 1$,

$$
\left\|\nu_{k} R^{2}\right\|_{\varepsilon} \lesssim\left\|\nu_{k}\right\|_{L^{\infty}(\mathbb{R})} \varepsilon^{-(d-1) / 3}\|R\|_{H_{\varepsilon}^{1}}^{2} \lesssim \varepsilon^{-(d-1) / 3}\|R\|_{H_{\varepsilon}^{1}}^{2},
$$

whereas for $k=0$,

$$
\left\|\nu_{0} R^{2}\right\|_{\varepsilon} \lesssim\left\|\nu_{0}\right\|_{L^{\infty}\left(J_{\varepsilon}\right)} \varepsilon^{-(d-1) / 3}\|R\|_{H_{\varepsilon}^{1}}^{2} \lesssim \varepsilon^{-d / 3}\|R\|_{H_{\varepsilon}^{1}}^{2}
$$

On the other side,

$$
\left\|R^{3}\right\|_{\varepsilon} \lesssim \varepsilon^{-2(d-1) / 3}\|R\|_{H_{\varepsilon}^{1}}^{3}
$$

thanks to Lemma 2.4 again. By Lemma 2.3 as well as bounds (2.25), (2.26), (2.29), (2.30) and $(2.31)$

$$
\left\|\Phi_{N, \varepsilon}(R)-R_{N, \varepsilon}^{0}\right\|_{H_{\varepsilon}^{1}} \lesssim \varepsilon^{2 / 3}+\varepsilon^{2 / 3}\|R\|_{H_{\varepsilon}^{1}}+\varepsilon^{(2 N+2-d) / 3}\|R\|_{H_{\varepsilon}^{1}}^{2}+\varepsilon^{(4 N+6-2 d) / 3}\|R\|_{H_{\varepsilon}^{1}}^{3},
$$

where

$$
R_{N, \varepsilon}^{0}:=\left(M^{\varepsilon}\right)^{-1} F_{N, 0} .
$$

In particular, for $\varepsilon>0$ sufficiently small and for some $\varepsilon$-independent constant $C>0, \Phi_{N, \varepsilon}$ maps the ball

$$
B_{\varepsilon}:=B_{H_{\varepsilon}^{1}}\left(R_{N, \varepsilon}^{0}, C \varepsilon^{2 / 3}\right)
$$

into itself, where we have used the assumption $N \geqslant 2$. Similarly, there exists an $\varepsilon$-independent constant $\tilde{C}>0$ such that for every $R_{1}, R_{2}$ in $B_{\varepsilon}$,

$$
\left\|\Phi_{N, \varepsilon}\left(R_{1}\right)-\Phi_{N, \varepsilon}\left(R_{2}\right)\right\|_{H_{\varepsilon}^{1}} \leqslant \tilde{C} \varepsilon^{2 / 3}\left\|R_{1}-R_{2}\right\|_{H_{\varepsilon}^{1}} .
$$


As a result, provided $\varepsilon$ is sufficiently small, $\Phi_{N, \varepsilon}$ is a contraction on $B_{\varepsilon}$. The Fixed Point Theorem ensures that $\Phi_{N, \varepsilon}$ has a unique fixed point $R_{N, \varepsilon} \in B_{\varepsilon}$. In particular,

$$
\left\|R_{N, \varepsilon}-R_{N, \varepsilon}^{0}\right\|_{H_{\varepsilon}^{1}} \lesssim \varepsilon^{2 / 3} .
$$

We next prove that $R_{N, \varepsilon}$ satisfies the regularity properties stated in Theorem 1 . The fixed point $R_{N, \varepsilon} \in H_{\varepsilon}^{1}$ of $\Phi_{N, \varepsilon}$ has been constructed in such a way that $T^{\varepsilon} R_{N, \varepsilon} \in H^{1}\left(\mathbb{R}^{d}\right)$ solves the equation

$$
K^{\varepsilon} T^{\varepsilon} R_{N, \varepsilon}=T^{\varepsilon}\left(F_{N, \varepsilon}\left(\cdot, R_{N, \varepsilon}\right)\right) \in L^{2}\left(\mathbb{R}^{d}\right) .
$$

Thanks to Lemma 2.3 and (2.25), we obtain

$$
\left\|R_{N, \varepsilon}^{0}\right\|_{H_{\varepsilon}^{1}} \lesssim\left\|F_{N, 0}\right\|_{\varepsilon} \lesssim 1
$$

Thus, (2.32) yields

$$
\left\|R_{N, \varepsilon}\right\|_{\varepsilon} \lesssim\left\|R_{N, \varepsilon}\right\|_{H_{\varepsilon}^{1}} \lesssim 1
$$

As a result, from $(2.25),(2.26),(2.29),(2.30)$ and $(2.31)$, we infer

$$
\left\|F_{N, \varepsilon}\left(\cdot, R_{N, \varepsilon}\right)\right\|_{\varepsilon} \lesssim 1
$$

¿From (2.33), (2.36) and (2.19) we deduce

$$
\left\|K^{\varepsilon} T^{\varepsilon} R_{N, \varepsilon}\right\|_{L^{2}\left(\mathbb{R}^{d}\right)}=\left\|T^{\varepsilon}\left(F_{N, \varepsilon}\left(\cdot, R_{N, \varepsilon}\right)\right)\right\|_{L^{2}\left(\mathbb{R}^{d}\right)} \lesssim \varepsilon^{-(d-1) / 3} .
$$

Next, we use the following Lemma, which is proved in Section 8.

Lemma $2.5\left(K^{\varepsilon}\right)^{-1} \in \mathcal{L}\left(L^{2}\left(\mathbb{R}^{d}\right), H^{2}\left(\mathbb{R}^{d}\right)\right)$ is uniformly bounded in $\varepsilon$.

As a result, we infer from the Sobolev embedding of $H^{2}\left(\mathbb{R}^{d}\right)$ into $L^{\infty}\left(\mathbb{R}^{d}\right)$ that $T^{\varepsilon} R_{N, \varepsilon} \in H^{2}\left(\mathbb{R}^{d}\right)$ and

$$
\left\|R_{N, \varepsilon}\right\|_{L^{\infty}\left(J_{\varepsilon}\right)}=\left\|T^{\varepsilon} R_{N, \varepsilon}\right\|_{L^{\infty}\left(\mathbb{R}^{d}\right)} \lesssim \varepsilon^{-(d-1) / 3} .
$$

Moreover, a bootstrapping argument shows that $T^{\varepsilon} R_{N, \varepsilon} \in \mathcal{C}^{\infty}\left(\mathbb{R}^{d}\right)$. As a result, $R_{N, \varepsilon} \in \mathcal{C}^{\infty}\left(J_{\varepsilon}\right)$.

\section{$2.3 \nu_{\varepsilon}(y)>0$ for all $y \in J_{\varepsilon}$}

We have constructed above $\left\{\nu_{n}\right\}_{n \geqslant 0}$ and $R_{N, \varepsilon}$ in such a way that

$$
\begin{aligned}
\tilde{\eta}_{\varepsilon}(x) & :=\varepsilon^{1 / 3} \sum_{n=0}^{N} \varepsilon^{2 n / 3} \nu_{n}\left(\frac{1-|x|^{2}}{\varepsilon^{2 / 3}}\right)+\varepsilon^{2 N / 3+1}\left(T^{\varepsilon} R_{N, \varepsilon}\right)\left(\varepsilon^{-2 / 3} x\right) \\
& =\varepsilon^{1 / 3} \nu_{\varepsilon}\left(\frac{1-|x|^{2}}{\varepsilon^{2 / 3}}\right), \quad x \in \mathbb{R}^{d}
\end{aligned}
$$

is a classical, radially symmetric solution of equation (2.1). In order to claim that $\tilde{\eta}_{\varepsilon}$ is a ground state, it is sufficient to check that $\tilde{\eta}_{\varepsilon}(x)>0$, for every $x \in \mathbb{R}^{d}$, which is equivalent to $\nu_{\varepsilon}(y)>0$, for every $y \in J_{\varepsilon}$. 
For every $n \geqslant 1,\left\|\nu_{n}\right\|_{L^{\infty}(\mathbb{R})} \lesssim 1$. Therefore, from (2.38), (2.39), since $N \geqslant 2$, we deduce the existence of a constant $C>0$ such that for every $y \in J_{\varepsilon}$,

$$
\nu_{\varepsilon}(y)-\nu_{0}(y) \geqslant-C \varepsilon^{2 / 3} .
$$

Since $\nu_{0}(y)$ increases from 0 to $+\infty$ as $y$ goes from $-\infty$ to $+\infty$, we deduce that for $\varepsilon \ll 1$,

$$
\nu_{\varepsilon}(y) \geqslant \nu_{0}(-1)-C \varepsilon^{2 / 3}>0, \quad y \in\left[-1, \varepsilon^{-2 / 3}\right] .
$$

Coming back to the variable $x$, it follows that

$$
\tilde{\eta}_{\varepsilon}(x)>0, \quad|x| \leqslant\left(1+\varepsilon^{2 / 3}\right)^{1 / 2} .
$$

It remains to prove that $\tilde{\eta}_{\varepsilon}(x)>0$ for all $|x|>\left(1+\varepsilon^{2 / 3}\right)^{1 / 2}$. Assume by contradiction that $\tilde{\eta}_{\varepsilon}$ is not strictly positive on $\mathbb{R}^{d}$. Then, let

$$
r_{\varepsilon}=\inf \left\{r>0, \tilde{\eta}_{\varepsilon}(r)=0\right\} \in\left(\left(1+\varepsilon^{2 / 3}\right)^{1 / 2}, \infty\right)
$$

where for convenience, since $\tilde{\eta}_{\varepsilon}$ is radial, we denote $\tilde{\eta}_{\varepsilon}(|x|)=\tilde{\eta}_{\varepsilon}(x)$. By construction, $\tilde{\eta}_{\varepsilon}\left(r_{\varepsilon}\right)=0$ and $\tilde{\eta}_{\varepsilon}^{\prime}\left(r_{\varepsilon}\right) \leqslant 0$. If $\tilde{\eta}_{\varepsilon}^{\prime}\left(r_{\varepsilon}\right)=0$, then $\tilde{\eta}_{\varepsilon} \equiv 0$, because $\tilde{\eta}_{\varepsilon}(r)$ satisfies the differential equation

$$
-\frac{1}{r^{d-1}} \frac{d}{d r}\left(r^{d-1} \frac{d}{d r} \tilde{\eta}_{\varepsilon}\right)(r)+\frac{1}{\varepsilon^{2}}\left(r^{2}-1+\tilde{\eta}_{\varepsilon}(r)^{2}\right) \tilde{\eta}_{\varepsilon}(r)=0 .
$$

This is a contradiction with $(2.40)$. Thus, $\tilde{\eta}_{\varepsilon}^{\prime}\left(r_{\varepsilon}\right)<0$. Let

$$
\tilde{r}_{\varepsilon}:=\sup \left\{r>r_{\varepsilon}, \tilde{\eta}_{\varepsilon}\left(r^{\prime}\right)<0 \text { for } r^{\prime} \in\left(r_{\varepsilon}, r\right)\right\} \in\left(r_{\varepsilon},+\infty\right] .
$$

Then, for every $r \in\left(r_{\varepsilon}, \tilde{r}_{\varepsilon}\right)$,

$$
\frac{d}{d r}\left(r^{d-1} \frac{d}{d r} \tilde{\eta}_{\varepsilon}\right)(r)=\frac{r^{d-1}}{\varepsilon^{2}}\left(r^{2}-1+\tilde{\eta}_{\varepsilon}(r)^{2}\right) \tilde{\eta}_{\varepsilon}(r) \leqslant 0,
$$

and we deduce by integration that for every $r \in\left(r_{\varepsilon}, \tilde{r}_{\varepsilon}\right)$,

$$
r^{d-1} \tilde{\eta}_{\varepsilon}^{\prime}(r) \leqslant r_{\varepsilon}^{d-1} \tilde{\eta}_{\varepsilon}^{\prime}\left(r_{\varepsilon}\right)<0
$$

and

$$
\tilde{\eta}_{\varepsilon}(r) \leqslant r_{\varepsilon}^{d-1} \tilde{\eta}_{\varepsilon}^{\prime}\left(r_{\varepsilon}\right) \int_{r_{\varepsilon}}^{r} s^{1-d} d s
$$

The right hand side in (2.41) is a negative, decreasing function of $r$, which implies $\tilde{r}_{\varepsilon}=+\infty$, as well as a contradiction with the fact that $\tilde{\eta}_{\varepsilon}(r) \rightarrow 0$ as $r \rightarrow+\infty$. Therefore $\tilde{\eta}_{\varepsilon}(r)>0$ for all $r \in \mathbb{R}_{+}$. 


\section{Spectrum of the Schrödinger operator $L_{+}^{\varepsilon}$ in the case $d=1$}

Consider the Schrödinger operator

$$
L_{+}^{\varepsilon}=-\varepsilon^{2} \partial_{x}^{2}+V_{\varepsilon}(x), \quad V_{\varepsilon}(x)=3 \eta_{\varepsilon}^{2}(x)-1+x^{2},
$$

associated with the stationary Gross-Pitaevskii equation (1.2) linearized at the ground state $\eta_{\varepsilon}$. It is a self-adjoint operator on $L^{2}(\mathbb{R})$. Since the potential $V_{\varepsilon}(x)$ is confining in the sense of $V_{\varepsilon}(x) \rightarrow+\infty$ as $|x| \rightarrow \infty, L_{+}^{\varepsilon}$ has compact resolvent and a purely discrete spectrum. By SturmLiouville theory, the eigenvalues of $L_{+}^{\varepsilon}$, denoted $\left\{\lambda_{n}^{\varepsilon}\right\}_{n \geqslant 1}$ (sorted in increasing order) are simple. Moreover, thanks to the even symmetry of $V_{\varepsilon}$ on $\mathbb{R}$, the eigenfunctions of $L_{+}^{\varepsilon}$ corresponding to $\lambda_{n}^{\varepsilon}$ are even (resp. odd) in $x$ if $n$ is odd (resp. even). If $\lambda$ is an eigenvalue of $L_{+}^{\varepsilon}$ and $\varphi \in L^{2}(\mathbb{R})$ is a corresponding eigenfunction, we define a function $v \in L_{\varepsilon}^{2}$ by

$$
\varphi(x)=v\left(\frac{1-x^{2}}{\varepsilon^{2 / 3}}\right), \quad x \in \mathbb{R}_{+} .
$$

Let us denote $W_{\varepsilon}(y)=3 \nu_{\varepsilon}^{2}(y)-y$. Then, $\varphi \in L^{2}(\mathbb{R})$ is an even eigenfunction of $L_{+}^{\varepsilon}$ corresponding to the eigenvalue $\lambda$ if and only if $v \in L_{\varepsilon}^{2}$ satisfies the differential equation

$$
\left(-4\left(1-\varepsilon^{2 / 3} y\right)^{1 / 2} \partial_{y}\left(1-\varepsilon^{2 / 3} y\right)^{1 / 2} \partial_{y}+W_{\varepsilon}(y)\right) v(y)=\varepsilon^{-2 / 3} \lambda v(y), \quad y \in J_{\varepsilon}
$$

and the Neumann boundary condition

$$
\varphi^{\prime}(0)=-\left.2 \varepsilon^{-2 / 3}\left(\left(1-\varepsilon^{2 / 3} y\right)^{1 / 2} v^{\prime}(y)\right)\right|_{y=\varepsilon^{-2 / 3}}=0 .
$$

Similarly, $\varphi \in L^{2}(\mathbb{R})$ is an odd eigenfunction of $L_{+}^{\varepsilon}$ corresponding to the eigenvalue $\lambda$ if and only if $v \in L_{\varepsilon}^{2}$ satisfies (3.1) and the Dirichlet boundary condition

$$
\varphi(0)=v\left(\varepsilon^{-2 / 3}\right)=0 .
$$

As a result, the eigenvalues of $L_{+}^{\varepsilon}$ are directly related to the eigenvalues of the two self-adjoint operators on $L_{\varepsilon}^{2}, \check{M}^{\varepsilon}$ and $\tilde{M}^{\varepsilon}$, where

$$
\left\{\begin{array}{l}
\check{M}^{\varepsilon}:=-4\left(1-\varepsilon^{2 / 3} y\right)^{1 / 2} \partial_{y}\left(1-\varepsilon^{2 / 3} y\right)^{1 / 2} \partial_{y}+W_{\varepsilon}(y) \\
\operatorname{Dom}\left(\check{M}^{\varepsilon}\right)=\left\{v \in L_{\varepsilon}^{2}: \check{M}^{\varepsilon} v \in L_{\varepsilon}^{2} \text { and } v \text { satisfies (NC) }\right\}
\end{array}\right.
$$

and $\tilde{M}^{\varepsilon}$ is defined similarly by replacing (NC) by (DC) in the definition of the domain. Namely, if we denote $\left\{\check{\mu}_{n}^{\varepsilon}\right\}_{n \geqslant 1}$ (resp. $\left\{\tilde{\mu}_{n}^{\varepsilon}\right\}_{n \geqslant 1}$ ) the eigenvalues of $\check{M}^{\varepsilon}$ (resp. $\tilde{M}^{\varepsilon}$ ) sorted in increasing order, then for every $n \geqslant 1$,

$$
\check{\mu}_{n}^{\varepsilon}=\varepsilon^{-2 / 3} \lambda_{2 n-1}^{\varepsilon} \quad \text { and } \quad \tilde{\mu}_{n}^{\varepsilon}=\varepsilon^{-2 / 3} \lambda_{2 n}^{\varepsilon} .
$$

As $\varepsilon \rightarrow 0$, the eigenvalue problems (3.1) for the operators $\check{M}^{\varepsilon}$ and $\tilde{M}^{\varepsilon}$ formally converge to the eigenvalue problem for the Schrödinger operator $M_{0}$ defined after Lemma 2.2,

$$
\left(-4 \partial_{y}^{2}+W_{0}(y)\right) v(y)=\mu v(y), \quad y \in \mathbb{R}, \quad \text { where } \mu=\varepsilon^{-2 / 3} \lambda .
$$

By the discussion below Lemma 2.2, the purely discrete spectrum of $M_{0}$ in $L^{2}(\mathbb{R})$ consists of an increasing sequence of positive eigenvalues $\left\{\mu_{n}\right\}_{n \geqslant 1}$. We shall prove that the eigenvalues of $L_{+}^{\varepsilon}$ converge to the eigenvalues of $M_{0}$ as $\varepsilon \rightarrow 0$, according to the following result. 
Theorem 2 The spectrum of $L_{+}^{\varepsilon}$ consists of an increasing sequence of positive eigenvalues $\left\{\lambda_{n}^{\varepsilon}\right\}_{n \geqslant 1}$ such that for each $n \geqslant 1$,

$$
\lim _{\varepsilon \downarrow 0} \frac{\lambda_{2 n-1}^{\varepsilon}}{\varepsilon^{2 / 3}}=\lim _{\varepsilon \downarrow 0} \frac{\lambda_{2 n}^{\varepsilon}}{\varepsilon^{2 / 3}}=\mu_{n}
$$

Proof. We prove only the convergence of $\tilde{\mu}_{n}^{\varepsilon}=\lambda_{2 n}^{\varepsilon} / \varepsilon^{2 / 3}$ to $\mu_{n}$, for every $n \geqslant 1$. The proof of the convergence of $\check{\mu}_{n}^{\varepsilon}=\lambda_{2 n-1}^{\varepsilon} / \varepsilon^{2 / 3}$ to $\mu_{n}$ is identical.

Denote by $\langle\cdot, \cdot\rangle$ and $\|\cdot\|$ the scalar product and the norm in $L^{2}(\mathbb{R})$, and by $\langle\cdot, \cdot\rangle_{\varepsilon}$ and $\|\cdot\|_{\varepsilon}$ the scalar product and the norm in $L_{\varepsilon}^{2}$. If $u, v \in L^{2}(\mathbb{R}), u \perp v$ means that $\langle u, v\rangle=0$, whereas if $u, v \in L_{\varepsilon}^{2}, u \perp_{\varepsilon} v$ means that $\langle u, v\rangle_{\varepsilon}=0$. We denote by

$$
R^{\varepsilon}(v)=\frac{Q^{\varepsilon}(v, v)}{\|v\|_{\varepsilon}^{2}}
$$

the Rayleigh quotient for the operator $\tilde{M}^{\varepsilon}$, where $Q^{\varepsilon}$ denotes the corresponding bilinear form

$$
Q^{\varepsilon}(u, v)=\int_{J_{\varepsilon}}\left(4\left(1-\varepsilon^{2 / 3} y\right)^{1 / 2} \partial_{y} u \partial_{y} v+\frac{W_{\varepsilon}(y)}{\left(1-\varepsilon^{2 / 3} y\right)^{1 / 2}} u(y) v(y)\right) d y,
$$

defined for $u, v \in H_{\varepsilon}^{1}$. Similarly,

$$
R(v)=\frac{Q(v, v)}{\|v\|^{2}}
$$

denotes the Rayleigh quotient for $M_{0}$, where $Q$ is the corresponding bilinear form

$$
Q(u, v)=\int_{\mathbb{R}}\left(4 \partial_{y} u \partial_{y} v+W_{0}(y) u(y) v(y)\right) d y,
$$

defined for $u, v \in\left\{u \in H^{1}(\mathbb{R}): W_{0}^{1 / 2} u \in L^{2}(\mathbb{R})\right\}$.

Let $\tilde{u}_{n}^{\varepsilon}$ (resp. $\left.u_{n}\right)$ denote an eigenfunction of $\tilde{M}^{\varepsilon}$ (resp. $M_{0}$ ) corresponding to the eigenvalue $\tilde{\mu}_{n}^{\varepsilon}\left(\right.$ resp. $\left.\mu_{n}\right)$, normalized by $\left\|\tilde{u}_{n}^{\varepsilon}\right\|_{\varepsilon}=1$ (resp. $\left\|u_{n}\right\|=1$ ). The eigenvalues of $M_{0}$ are given by the Max-Min principle:

$$
\mu_{n}=\inf _{\substack{v \in \operatorname{Dom}\left(M_{0}\right) \\ v \perp u_{1}, \cdots, u_{n-1}}} R(v),
$$

whereas the eigenvalues of $\tilde{M}^{\varepsilon}$ are similarly given by

$$
\tilde{\mu}_{n}^{\varepsilon=} \underset{\substack{v \in \operatorname{Dom}\left(\tilde{M}^{\varepsilon}\right) \\ v \perp_{\varepsilon} \tilde{u}_{1}^{\varepsilon}, \cdots, \tilde{u}_{n-1}^{\varepsilon}}}{R} R^{\varepsilon}(v) .
$$

Let us fix $\delta \in(0,2 / 3)$. Let $\Phi \in \mathcal{C}^{\infty}(\mathbb{R})$ be an non-decreasing function such that $\Phi \equiv 0$ on $\mathbb{R}_{-}$ and $\Phi \equiv 1$ on $[1,+\infty)$. For $\varepsilon>0$ sufficiently small, we also define $\chi_{\varepsilon} \in \mathcal{C}_{c}^{\infty}(\mathbb{R})$ by

$$
\chi_{\varepsilon}(x)=\Phi\left(\frac{2 x+\varepsilon^{-2 / 3}}{\varepsilon^{-2 / 3}-2 \varepsilon^{-\delta}}\right) \Phi\left(\frac{\varepsilon^{-2 / 3}-2 x}{\varepsilon^{-2 / 3}-2 \varepsilon^{-\delta}}\right),
$$


such that $\chi_{\varepsilon}$ is even, $\chi_{\varepsilon} \equiv 1$ on $\left[-\varepsilon^{-\delta}, \varepsilon^{-\delta}\right]$ and $\operatorname{Supp}\left(\chi_{\varepsilon}\right) \subset\left[-\frac{\varepsilon^{-2 / 3}}{2}, \frac{\varepsilon^{-2 / 3}}{2}\right]$. We shall prove recursively the following properties:

$$
\left\{\begin{array}{l}
(\mathrm{i})_{\mathrm{n}} \tilde{\mu}_{n}^{\varepsilon}=\mu_{n}+\mathcal{O}\left(\varepsilon^{2 / 3-\delta}\right), \\
(\text { ii })_{\mathrm{n}} \text { for every } k \geqslant n+1,\left\langle\chi_{\varepsilon} u_{k}, \tilde{u}_{n}^{\varepsilon}\right\rangle_{\varepsilon}=\mathcal{O}\left(\varepsilon^{1 / 3-\delta / 2}\right), \\
(\text { iii) })_{\mathrm{n}} \text { for every } k \geqslant n,\left\langle\chi_{\varepsilon} \tilde{u}_{k}^{\varepsilon}, u_{n-1}\right\rangle=\mathcal{O}\left(\varepsilon^{1 / 3-\delta / 2}\right), \\
\text { (iv) } \inf _{c \in \mathbb{R}}\left\|\chi_{\varepsilon} \tilde{u}_{n}^{\varepsilon}-c u_{n}\right\|=\mathcal{O}\left(\varepsilon^{1 / 3-\delta / 2}\right), \\
(\mathrm{v})_{\mathrm{n}} \inf _{c \in \mathbb{R}}\left\|\chi_{\varepsilon} u_{n-1}-c \tilde{u}_{n-1}^{\varepsilon}\right\|_{\varepsilon}=\mathcal{O}\left(\varepsilon^{1 / 3-\delta / 2}\right),
\end{array}\right.
$$

where for $n=1$, (iii) $)_{1}$ and $(\mathrm{v})_{1}$ have to be understood as empty properties. Let us fix $n \geqslant 1$ and assume that $\left(\mathrm{G}_{\mathrm{k}}\right)$ is true for every $k \in\{1, \cdots, n-1\}$ (for $n=1$, this condition is empty, therefore true by convention). The proof of $\left(\mathrm{G}_{\mathrm{n}}\right)$ is then divided in five steps.

Step 1. Upper bound on $\tilde{\mu}_{n}^{\varepsilon}$. First, we shall prove that

$$
R^{\varepsilon}\left(v_{n}^{\varepsilon}\right)=\mu_{n}+\mathcal{O}\left(\varepsilon^{2 / 3-\delta}\right), \text { where } v_{n}^{\varepsilon}=\chi_{\varepsilon} u_{n}-\sum_{k=1}^{n-1}\left\langle\chi_{\varepsilon} u_{n}, \tilde{u}_{k}^{\varepsilon}\right\rangle_{\varepsilon} \tilde{u}_{k}^{\varepsilon} .
$$

Then, thanks to (3.4), since $v_{n}^{\varepsilon} \in \operatorname{Span}\left(\tilde{u}_{1}^{\varepsilon}, \cdots, \tilde{u}_{n-1}^{\varepsilon}\right)^{\perp_{\varepsilon}} \subset L_{\varepsilon}^{2}$ by construction, (3.5) yields

$$
\tilde{\mu}_{n}^{\varepsilon} \leqslant \mu_{n}+\mathcal{O}\left(\varepsilon^{2 / 3-\delta}\right) .
$$

¿From $(\mathrm{i})_{\mathrm{k}}$ and $(\mathrm{ii})_{\mathrm{k}}$, which are satisfied for $k \leqslant n-1$ thanks to the recursion assumption, we have

$$
R^{\varepsilon}\left(v_{n}^{\varepsilon}\right)=\frac{Q^{\varepsilon}\left(\chi_{\varepsilon} u_{n}, \chi_{\varepsilon} u_{n}\right)-\sum_{k=1}^{n-1} \tilde{\mu}_{k}^{\varepsilon}\left\langle\chi_{\varepsilon} u_{n}, \tilde{u}_{k}^{\varepsilon}\right\rangle_{\varepsilon}^{2}}{\left\|\chi_{\varepsilon} u_{n}\right\|_{\varepsilon}^{2}-\sum_{k=1}^{n-1}\left\langle\chi_{\varepsilon} u_{n}, \tilde{u}_{k}^{\varepsilon}\right\rangle_{\varepsilon}^{2}}=\frac{Q^{\varepsilon}\left(\chi_{\varepsilon} u_{n}, \chi_{\varepsilon} u_{n}\right)+\mathcal{O}\left(\varepsilon^{2 / 3-\delta}\right)}{\left\|\chi_{\varepsilon} u_{n}\right\|_{\varepsilon}^{2}+\mathcal{O}\left(\varepsilon^{2 / 3-\delta}\right)} .
$$

Next,

$$
\begin{aligned}
\left\|\chi_{\varepsilon} u_{n}\right\|_{\varepsilon}^{2} & =\int_{-\frac{\varepsilon^{-2 / 3}}{2}}^{\frac{\varepsilon^{-2 / 3}}{2}} \frac{\chi_{\varepsilon}^{2} u_{n}^{2}}{\left(1-\varepsilon^{2 / 3} y\right)^{1 / 2}} d y \\
& =\left(1+\mathcal{O}\left(\varepsilon^{2 / 3-\delta}\right)\right) \int_{-\varepsilon^{-\delta}}^{\varepsilon^{-\delta}} u_{n}^{2} d y+\int_{\varepsilon^{-\delta} \leqslant|y| \leqslant \frac{\varepsilon^{-2 / 3}}{2}} \frac{\chi_{\varepsilon}^{2} u_{n}^{2}}{\left(1-\varepsilon^{2 / 3} y\right)^{1 / 2}} d y .
\end{aligned}
$$

The last term in the right hand side of (3.8) is estimated as follows

$$
\int_{\varepsilon^{-\delta} \leqslant|y| \leqslant \frac{\varepsilon^{-2 / 3}}{2}} \frac{\chi_{\varepsilon}^{2} u_{n}^{2}}{\left(1-\varepsilon^{2 / 3} y\right)^{1 / 2}} d y \leqslant \sqrt{2} \int_{|y| \geqslant \varepsilon^{-\delta}} u_{n}^{2} d y \lesssim \exp \left(-2 \varepsilon^{-\delta}\right) \lesssim \varepsilon^{2 / 3},
$$

where we have used the following Lemma. 
Lemma 3.1 For every $m \geqslant 1$, there exists a constant $C_{m}>0$ such that for every $y \in \mathbb{R}$,

$$
\left|u_{m}(y)\right| \leqslant C_{m} \exp (-|y|)
$$

and

$$
\left|u_{m}^{\prime}(y)\right| \leqslant C_{m}(|y|+1) \exp (-|y|) .
$$

Proof. Since $W_{0}(y) \rightarrow+\infty$ as $y \rightarrow \infty$, we can fix $b_{n}>0$ such that $\inf \left\{W_{0}(y):|y| \geqslant b_{n}\right\}>$ $4+\mu_{n}$. Then,

$$
\left(-4 \partial_{y}^{2}+W_{0}(y)-\mu_{n}\right) e^{-|y|}=\left(W_{0}(y)-\mu_{n}-4\right) e^{-|y|} \geqslant 0, \quad|y|>b_{n} .
$$

Since $u_{n}$ solves the eigenvalue problem

$$
\left(-4 \partial_{y}^{2}+W_{0}(y)-\mu_{n}\right) u_{n}=0, \quad y \in \mathbb{R}
$$

thanks to Corollary 2.8 in [A], there exists $C>0$ such that

$$
\left|u_{n}(y)\right| \leqslant C e^{-|y|}, \quad|y| \geqslant b_{n}+1 .
$$

Bound (3.10) follows, since $u_{n} \in \operatorname{Dom}\left(M_{0}\right) \subset H^{1}(\mathbb{R}) \subset L^{\infty}(\mathbb{R})$. Then, from the differential equation $M_{0} u_{n}=\mu_{n} u_{n}$ and thanks to the asymptotic behaviour of $W_{0}$, we infer

$$
\left|u_{n}^{\prime \prime}(y)\right|=\frac{1}{4}\left|\mu_{n} u_{n}(y)-W_{0}(y) u_{n}(y)\right| \lesssim(|y|+1) e^{-|y|}, \quad y \in \mathbb{R} .
$$

By integration of (3.12) between $-\infty$ and $y$, we deduce, for $y<0$,

$$
\left|u_{n}^{\prime}(y)\right|=\left|\int_{-\infty}^{y} u_{n}^{\prime \prime}(s) d s\right| \lesssim(|y|+1) e^{-|y|} .
$$

The same kind of estimate is obtained for $y>0$ by integration of (3.12) between $y$ and $+\infty$, which provides (3.11) and completes the proof of Lemma 3.1.

Using Lemma 3.1 again, as well as the normalization of $u_{n}$, we infer that

$$
\int_{-\varepsilon^{-\delta}}^{\varepsilon^{-\delta}} u_{n}^{2} d y=1+\mathcal{O}\left(\varepsilon^{2 / 3}\right)
$$

¿From (3.8), (3.9) and (3.13), we deduce that

$$
\left\|\chi_{\varepsilon} u_{n}\right\|_{\varepsilon}^{2}=1+\mathcal{O}\left(\varepsilon^{2 / 3-\delta}\right) .
$$

On the other side,

$$
\begin{aligned}
Q^{\varepsilon}\left(\chi_{\varepsilon} u_{n}, \chi_{\varepsilon} u_{n}\right)= & \int_{J_{\varepsilon}}\left[4\left(1-\varepsilon^{2 / 3} y\right)^{1 / 2}\left|\partial_{y}\left(\chi_{\varepsilon} u_{n}\right)\right|^{2}+\frac{W_{\varepsilon}\left|\chi_{\varepsilon} u_{n}\right|^{2}}{\left(1-\varepsilon^{2 / 3} y\right)^{1 / 2}}\right] d y \\
= & 4 \int_{J_{\varepsilon}}\left(1-\varepsilon^{2 / 3} y\right)^{1 / 2} \chi_{\varepsilon}^{\prime 2} u_{n}^{2} d y+8 \int_{J_{\varepsilon}}\left(1-\varepsilon^{2 / 3} y\right)^{1 / 2} \chi_{\varepsilon}^{\prime} \chi_{\varepsilon} u_{n}^{\prime} u_{n} d y \\
& +4 \int_{-\varepsilon^{-\delta}}^{\varepsilon^{-\delta}}\left(1-\varepsilon^{2 / 3} y\right)^{1 / 2} u_{n}^{\prime 2} d y+4 \int_{\varepsilon^{-\delta} \leqslant|y| \leqslant \frac{\varepsilon^{-2 / 3}}{2}}\left(1-\varepsilon^{2 / 3} y\right)^{1 / 2} \chi_{\varepsilon}^{2} u_{n}^{\prime 2} d y \\
& +\int_{-\varepsilon^{-\delta / 2}}^{\varepsilon^{-\delta / 2}} \frac{W_{\varepsilon} u_{n}^{2}}{\left(1-\varepsilon^{2 / 3} y\right)^{1 / 2}} d y+\int_{\varepsilon^{-\delta / 2} \leqslant|y| \leqslant \frac{\varepsilon^{-2 / 3}}{2}} \frac{W_{\varepsilon}\left|\chi_{\varepsilon} u_{n}\right|^{2}}{\left(1-\varepsilon^{2 / 3} y\right)^{1 / 2}} d y .
\end{aligned}
$$


The first two integrals in the right hand side of $(3.15)$ are $\mathcal{O}\left(\varepsilon^{2 / 3}\right)$, because $u_{n} \in H^{1}(\mathbb{R})$,

$$
\left\|\chi_{\varepsilon}^{\prime}\right\|_{L^{\infty}\left(J_{\varepsilon}\right)} \lesssim \varepsilon^{2 / 3}
$$

and

$$
\max \left\{\left(1-\varepsilon^{2 / 3} y\right)^{1 / 2}: y \in \operatorname{Supp} \chi_{\varepsilon}\right\} \leqslant \sqrt{3 / 2}
$$

The fourth and last integrals in the right hand side of $(3.15)$ are also $\mathcal{O}\left(\varepsilon^{2 / 3}\right)$, thanks to Lemma 3.1. From Lemma 3.1, we also infer that

$$
\int_{-\varepsilon^{-\delta}}^{\varepsilon^{-\delta}}\left(1-\varepsilon^{2 / 3} y\right)^{1 / 2} u_{n}^{\prime 2} d y=\left(1+\mathcal{O}\left(\varepsilon^{2 / 3-\delta}\right)\right) \int_{-\varepsilon^{-\delta}}^{\varepsilon^{-\delta}} u_{n}^{\prime 2} d y=\int_{-\infty}^{+\infty} u_{n}^{\prime 2} d y+\mathcal{O}\left(\varepsilon^{2 / 3-\delta}\right) .
$$

¿From Theorem 1 and from the decay properties of the function $\nu_{n}$ for $n \geqslant 1$ provided in $\left(\mathrm{H}_{\mathrm{n}}\right)$, we deduce that $\nu_{\varepsilon}=\nu_{0}+\varepsilon^{2 / 3} r_{\varepsilon}$, where $r_{\varepsilon}=\mathcal{O}_{L^{\infty}(\mathbb{R})}(1)$ and $\nu_{0} r_{\varepsilon}=\mathcal{O}_{L^{\infty}(\mathbb{R})}(1)$ as $\varepsilon \rightarrow 0$. As a result, $W_{\varepsilon}-W_{0}=3\left(\nu_{\varepsilon}^{2}-\nu_{0}^{2}\right) \in L^{\infty}(\mathbb{R})$, and

$$
\left\|W_{\varepsilon}-W_{0}\right\|_{L^{\infty}(\mathbb{R})} \lesssim \varepsilon^{2 / 3} .
$$

Then, since $W_{0}(y)=\mathcal{O}(y)$ as $y \rightarrow \pm \infty$,

$$
\left\|\frac{W_{\varepsilon}}{\left(1-\varepsilon^{2 / 3} y\right)^{1 / 2}}-W_{0}\right\|_{L^{\infty}\left(-\varepsilon^{-\delta / 2}, \varepsilon^{-\delta / 2}\right)} \lesssim \varepsilon^{2 / 3-\delta} .
$$

As a result, using once more Lemma 3.1,

$$
\int_{-\varepsilon^{-\delta / 2}}^{\varepsilon^{-\delta / 2}} \frac{W_{\varepsilon} u_{n}^{2}}{\left(1-\varepsilon^{2 / 3} y\right)^{1 / 2}} d y=\int_{-\infty}^{+\infty} W_{0} u_{n}^{2} d y+\mathcal{O}\left(\varepsilon^{2 / 3-\delta}\right) .
$$

Finally, we get from (3.7), (3.14), (3.15), (3.16), (3.19) and the estimates on the other term in the right hand side of $(3.15)$ :

$$
R^{\varepsilon}\left(v_{n}^{\varepsilon}\right)=R\left(u_{n}\right)+\mathcal{O}\left(\varepsilon^{2 / 3-\delta}\right)=\mu_{n}+\mathcal{O}\left(\varepsilon^{2 / 3-\delta}\right),
$$

which completes the proof of (3.5) and of its corollary (3.6).

Step 2. Asymptotic behaviour of the eigenfunction $\tilde{u}_{n}^{\varepsilon}$. Property $(\mathrm{i})_{\mathrm{n}}$ will be obtained as a consequence of (3.6) and of the converse inequality

$$
\mu_{n} \leqslant \tilde{\mu}_{n}^{\varepsilon}+\mathcal{O}\left(\varepsilon^{2 / 3-\delta}\right) .
$$

The proof of the latter inequality is delivered in Step 3 below. The proof uses the following properties of the eigenfunction $\tilde{u}_{n}^{\varepsilon}$ corresponding to the $n^{\text {th }}$ eigenvalue $\tilde{\mu}_{n}^{\varepsilon}$ of $\tilde{M}_{\varepsilon}$.

Lemma 3.2 There exists a constant $\tilde{C}_{n}>0$ such that for every $y \in J_{\varepsilon}$ and $\varepsilon>0$ sufficiently small,

$$
\left|\tilde{u}_{n}^{\varepsilon}(y)\right| \leqslant \tilde{C}_{n} e^{-|y|}
$$

whereas

$$
\left|\left(\tilde{u}_{n}^{\varepsilon}\right)^{\prime}(y)\right| \leqslant \begin{cases}\tilde{C}_{n}(|y|+1) e^{-|y|} & \text { if } y \leqslant 0, \\ \tilde{C}_{n}(|y|+1) e^{-|y|}+\exp \left(-\frac{\varepsilon^{-2 / 3}}{4}\right) & \text { if } 0<y \leqslant \frac{\varepsilon^{-2 / 3}}{2}, \\ \frac{\tilde{C}_{n} \exp \left(-\frac{\varepsilon^{-2 / 3}}{4}\right)}{\left(1-\varepsilon^{2 / 3} y\right)^{1 / 2}} & \text { if } \quad \frac{\varepsilon^{-2 / 3}}{2}<y \leqslant \varepsilon^{-2 / 3} .\end{cases}
$$


Proof. In order to prove (3.21), we come back to the eigenfunction

$$
\varphi_{2 n}^{\varepsilon}(x)=\tilde{u}_{n}^{\varepsilon}\left(\frac{1-x^{2}}{\varepsilon^{2 / 3}}\right)
$$

of $L_{+}^{\varepsilon}$ corresponding to the eigenvalue $\lambda_{2 n}^{\varepsilon}=\tilde{\mu}_{n}^{\varepsilon} \varepsilon^{2 / 3}$. Since

$$
\left\|\tilde{u}_{n}^{\varepsilon}\right\|_{H_{\varepsilon}^{1}}^{2}=Q^{\varepsilon}\left(\tilde{u}_{n}^{\varepsilon}, \tilde{u}_{n}^{\varepsilon}\right)=\tilde{\mu}_{n}^{\varepsilon}\left\|\tilde{u}_{n}^{\varepsilon}\right\|_{\varepsilon}^{2}=\tilde{\mu}_{n}^{\varepsilon},
$$

it follows from (3.6) and Lemma 2.4 that for $\varepsilon$ sufficiently small,

$$
\left\|\varphi_{2 n}^{\varepsilon}\right\|_{L^{\infty}(\mathbb{R})}=\left\|\tilde{u}_{n}^{\varepsilon}\right\|_{L^{\infty}\left(J_{\varepsilon}\right)} \leqslant C \sqrt{\tilde{\mu}_{n}^{\varepsilon}} \leqslant C \sqrt{\mu_{n}+\mathcal{O}\left(\varepsilon^{2 / 3-\delta}\right)} \leqslant c_{n},
$$

where $c_{n}>0$ is an $\varepsilon$-independent constant. Since $W_{0}(y) \gtrsim|y|$ as $y \rightarrow \pm \infty$, we can fix $a_{n}$ large enough such that $\inf \left\{W_{0}(y):|y| \geqslant a_{n}\right\}>4+\mu_{n}$. Then, using (3.17) and (3.6), we obtain, for $x^{2}<1-a_{n} \varepsilon^{2 / 3}$ and for $\varepsilon$ small enough,

$$
\begin{aligned}
& \left(-\varepsilon^{2} \partial_{x}^{2}+x^{2}-1+3 \eta_{\varepsilon}^{2}-\varepsilon^{2 / 3} \tilde{\mu}_{n}^{\varepsilon}\right) \exp \left(-\frac{1-x^{2}}{\varepsilon^{2 / 3}}\right) \\
& \quad=\varepsilon^{2 / 3}\left(-2 \varepsilon^{2 / 3}-4 x^{2}+W_{\varepsilon}\left(\frac{1-x^{2}}{\varepsilon^{2 / 3}}\right)-\tilde{\mu}_{n}^{\varepsilon}\right) \exp \left(-\frac{1-x^{2}}{\varepsilon^{2 / 3}}\right) \\
& \geqslant \varepsilon^{2 / 3}\left(-4+\inf \left\{W_{0}(y): y \geqslant a_{n}\right\}-\mu_{n}+\mathcal{O}\left(\varepsilon^{2 / 3-\delta}\right)\right) \exp \left(-\frac{1-x^{2}}{\varepsilon^{2 / 3}}\right) \geqslant 0 .
\end{aligned}
$$

On the other side, $\varphi_{2 n}^{\varepsilon}$ solves the differential equation

$$
\left(-\varepsilon^{2} \partial_{x}^{2}+x^{2}-1+3 \eta_{\varepsilon}^{2}-\varepsilon^{2 / 3} \tilde{\mu}_{n}^{\varepsilon}\right) \varphi_{2 n}^{\varepsilon}=0 .
$$

Thus,

$$
\left(-\varepsilon^{2} \partial_{x}^{2}+x^{2}-1+3 \eta_{\varepsilon}^{2}-\varepsilon^{2 / 3} \tilde{\mu}_{n}^{\varepsilon}\right) \psi_{n \pm}^{\varepsilon} \geqslant 0, \quad|x|<\left(1-a_{n} \varepsilon^{2 / 3}\right)^{1 / 2}
$$

where

$$
\psi_{n \pm}^{\varepsilon}(x)=c_{n} \exp \left(a_{n}-\frac{1-x^{2}}{\varepsilon^{2 / 3}}\right) \pm \varphi_{2 n}^{\varepsilon}(x) .
$$

Moreover, from (3.23), we get

$$
\psi_{n \pm}^{\varepsilon}\left( \pm\left(1-a_{n} \varepsilon^{2 / 3}\right)^{1 / 2}\right) \geqslant 0 .
$$

As a result, since for $\varepsilon$ small enough, we also have like in (3.24)

$$
\begin{aligned}
x^{2}-1+3 \eta_{\varepsilon}^{2}-\varepsilon^{2 / 3} \tilde{\mu}_{n}^{\varepsilon} & =\varepsilon^{2 / 3}\left(W_{\varepsilon}\left(\frac{1-x^{2}}{\varepsilon^{2 / 3}}\right)-\tilde{\mu}_{n}^{\varepsilon}\right) \\
& \geqslant \varepsilon^{2 / 3}\left(\inf \left\{W_{0}(y): y \geqslant a_{n}\right\}-\mu_{n}+\mathcal{O}\left(\varepsilon^{2 / 3-\delta}\right)\right)>0
\end{aligned}
$$

the maximum principle ensures that

$$
\psi_{n \pm}^{\varepsilon}(x) \geqslant 0, \quad|x|<\left(1-a_{n} \varepsilon^{2 / 3}\right)^{1 / 2},
$$


which is equivalent to

$$
\left|\varphi_{2 n}^{\varepsilon}(x)\right| \leqslant c_{n} \exp \left(a_{n}-\frac{1-x^{2}}{\varepsilon^{2 / 3}}\right), \quad|x|<\left(1-a_{n} \varepsilon^{2 / 3}\right)^{1 / 2} .
$$

In terms of $\tilde{u}_{n}^{\varepsilon}$, it means that

$$
\left|\tilde{u}_{n}^{\varepsilon}(y)\right| \leqslant c_{n} e^{a_{n}} e^{-y}, \quad a_{n} \leqslant y \leqslant \varepsilon^{-2 / 3} .
$$

On the other side, for $|x| \geqslant\left(1+a_{n} \varepsilon^{2 / 3}\right)^{1 / 2}$ and for $\varepsilon$ sufficiently small, we obtain like in (3.24)

$$
\begin{aligned}
( & \left.-\varepsilon^{2} \partial_{x}^{2}+x^{2}-1+3 \eta_{\varepsilon}^{2}-\varepsilon^{2 / 3} \tilde{\mu}_{n}^{\varepsilon}\right) \exp \left(-\frac{x^{2}-1}{\varepsilon^{2 / 3}}\right) \\
& =\varepsilon^{2 / 3}\left(2 \varepsilon^{2 / 3}-4 x^{2}+W_{\varepsilon}\left(\frac{1-x^{2}}{\varepsilon^{2 / 3}}\right)-\tilde{\mu}_{n}^{\varepsilon}\right) \exp \left(-\frac{x^{2}-1}{\varepsilon^{2 / 3}}\right) \\
& \geqslant \varepsilon^{2 / 3}\left(-4+W_{0}\left(\frac{1-x^{2}}{\varepsilon^{2 / 3}}\right)\left(4 \varepsilon^{2 / 3} \frac{\frac{1-x^{2}}{\varepsilon^{2 / 3}}}{W_{0}\left(\frac{1-x^{2}}{\varepsilon^{2 / 3}}\right)}+\frac{W_{\varepsilon}\left(\frac{1-x^{2}}{\varepsilon^{2 / 3}}\right)}{W_{0}\left(\frac{1-x^{2}}{\varepsilon^{2 / 3}}\right)}\right)-\mu_{n}+\mathcal{O}\left(\varepsilon^{2 / 3}\right)\right) \exp \left(-\frac{x^{2}-1}{\varepsilon^{2 / 3}}\right) \\
& \geqslant 0 .
\end{aligned}
$$

Thus, $\exp \left(-\frac{x^{2}-1}{\varepsilon^{2 / 3}}\right)$ is a positive, continuous supersolution of

$$
\left(-\varepsilon^{2} \partial_{x}^{2}+x^{2}-1+3 \eta_{\varepsilon}^{2}-\varepsilon^{2 / 3} \tilde{\mu}_{n}^{\varepsilon}\right) \varphi=0
$$

in $\left\{x:|x|>\left(1+a_{n} \varepsilon^{2 / 3}\right)^{1 / 2}\right\}$. From a slightly modified version of Corollary 2.8 in [A], we deduce that

$$
\left|\varphi_{2 n}^{\varepsilon}(x)\right| \leqslant 2 c_{n} \exp \left(1+a_{n}-\frac{x^{2}-1}{\varepsilon^{2 / 3}}\right), \quad|x| \geqslant\left(1+\left(a_{n}+1\right) \varepsilon^{2 / 3}\right)^{1 / 2} .
$$

More precisely, the constant $2 c_{n} e^{\left(a_{n}+1\right)}$ above has been chosen in such a way that the inequality holds for $|x|=\left(1+\left(a_{n}+1\right) \varepsilon^{2 / 3}\right)^{1 / 2}$, and the result in $[\mathrm{A}]$ ensures that then, the inequality holds for any $x$ such that $|x| \geqslant\left(1+\left(a_{n}+1\right) \varepsilon^{2 / 3}\right)^{1 / 2}$. In terms of $\tilde{u}_{n}^{\varepsilon}$, it means that

$$
\left|\tilde{u}_{n}^{\varepsilon}(y)\right| \leqslant 2 c_{n} e^{a_{n}+1} e^{y}, \quad y \leqslant-\left(a_{n}+1\right) .
$$

Then, (3.21) follows from (3.23), (3.28) and (3.30). We next prove (3.22). From (3.21) and the differential equation $\tilde{M}^{\varepsilon} \tilde{u}_{n}^{\varepsilon}=\tilde{\mu}_{n}^{\varepsilon} \tilde{u}_{n}^{\varepsilon}$, we infer that for every $y \in J_{\varepsilon}$,

$$
\left|\left(\partial_{y}\left(1-\varepsilon^{2 / 3} y\right)^{1 / 2} \partial_{y} \tilde{u}_{n}^{\varepsilon}\right)(y)\right| \leqslant \frac{\tilde{C}_{n} e^{-|y|}}{4\left(1-\varepsilon^{2 / 3} y\right)^{1 / 2}}\left(\mu_{n}+\left(\sup _{y \in \mathbb{R}} \frac{W_{0}(y)}{|y|}\right)|y|+\mathcal{O}\left(\varepsilon^{2 / 3-\delta}\right)\right),
$$

where we have also used (3.6) and (3.17). The estimate (3.22) in the case $y<0$ directly follows by integration of (3.31) between $-\infty$ and $y$ :

$$
\left|\left(\tilde{u}_{n}^{\varepsilon}\right)^{\prime}(y)\right| \leqslant\left|\left(1-\varepsilon^{2 / 3} y\right)^{1 / 2}\left(\tilde{u}_{n}^{\varepsilon}\right)^{\prime}(y)\right|=\left|\int_{-\infty}^{y}\left(\partial_{y}\left(1-\varepsilon^{2 / 3} y\right)^{1 / 2} \partial_{y} \tilde{u}_{n}^{\varepsilon}\right)(s) d s\right| \lesssim(|y|+1) e^{-|y|}
$$


As for the case $0<y<\frac{\varepsilon^{-2 / 3}}{2}$, integration of (3.31) between $y$ and $\frac{\varepsilon^{-2 / 3}}{2}$ gives

$$
\left|\left(1-\varepsilon^{2 / 3} y\right)^{1 / 2}\left(\tilde{u}_{n}^{\varepsilon}\right)^{\prime}(y)-\frac{1}{\sqrt{2}}\left(\tilde{u}_{n}^{\varepsilon}\right)^{\prime}\left(\frac{\varepsilon^{-2 / 3}}{2}\right)\right| \lesssim(|y|+1) e^{-|y|},
$$

which provides thanks to the triangular inequality

$$
\left|\left(\tilde{u}_{n}^{\varepsilon}\right)^{\prime}(y)\right| \lesssim(|y|+1) e^{-|y|}+\left|\left(\tilde{u}_{n}^{\varepsilon}\right)^{\prime}\left(\frac{\varepsilon^{-2 / 3}}{2}\right)\right| .
$$

Using basic integration, we also have

$$
\begin{aligned}
& \tilde{u}_{n}^{\varepsilon}\left(\frac{\varepsilon^{-2 / 3}}{2}\right)-\tilde{u}_{n}^{\varepsilon}\left(\frac{\varepsilon^{-2 / 3}}{4}\right) \\
& \quad=\int_{\frac{\varepsilon^{-2 / 3}}{4}}^{\frac{\varepsilon^{-2 / 3}}{2}}\left(\left(\tilde{u}_{n}^{\varepsilon}\right)^{\prime}(s)-\frac{\left(\tilde{u}_{n}^{\varepsilon}\right)^{\prime}\left(\frac{\varepsilon^{-2 / 3}}{2}\right)}{\sqrt{2}\left(1-\varepsilon^{2 / 3} s\right)^{1 / 2}}\right) d s+\frac{\left(\tilde{u}_{n}^{\varepsilon}\right)^{\prime}\left(\frac{\varepsilon^{-2 / 3}}{2}\right)}{\sqrt{2}} \int_{\frac{\varepsilon^{-2 / 3}}{4}}^{\frac{\varepsilon^{-2 / 3}}{2}} \frac{1}{\left(1-\varepsilon^{2 / 3} s\right)^{1 / 2}} d s .
\end{aligned}
$$

Since the last integral in the right hand side of $(3.35)$ is bounded from below by $\frac{\varepsilon^{-2 / 3} \sqrt{2}}{4}$, we deduce from (3.35), (3.33) and (3.21) that

$$
\left|\left(\tilde{u}_{n}^{\varepsilon}\right)^{\prime}\left(\frac{\varepsilon^{-2 / 3}}{2}\right)\right| \lesssim \exp \left(-\frac{\varepsilon^{-2 / 3}}{4}\right) .
$$

Combining (3.36) and (3.34), we get (3.22) in the case when $0<y<\frac{\varepsilon^{-2 / 3}}{2}$. Finally, we consider the case when $\frac{\varepsilon^{-2 / 3}}{2}<y<\varepsilon^{-2 / 3}$. Integration of (3.31) between $\frac{\varepsilon^{-2 / 3}}{2}$ and $y$ yields

$$
\begin{aligned}
\left|\left(\tilde{u}_{n}^{\varepsilon}\right)^{\prime}(y)\right| & \lesssim \frac{1}{\left(1-\varepsilon^{2 / 3} y\right)^{1 / 2}}\left(\sqrt{\frac{1}{2}}\left|\left(\tilde{u}_{n}^{\varepsilon}\right)^{\prime}\left(\frac{\varepsilon^{-2 / 3}}{2}\right)\right|+\int_{\frac{\varepsilon^{-2 / 3}}{2}}^{y} \frac{(|s|+1) e^{-|s|}}{\left(1-\varepsilon^{2 / 3} s\right)^{1 / 2}} d s\right) \\
& \lesssim \frac{1}{\left(1-\varepsilon^{2 / 3} y\right)^{1 / 2}}\left(\exp \left(-\frac{\varepsilon^{-2 / 3}}{4}\right)+\varepsilon^{-2 / 3} \exp \left(-\frac{\varepsilon^{-2 / 3}}{2}\right) \int_{\frac{\varepsilon^{-2 / 3}}{2}}^{\varepsilon^{-2 / 3}} \frac{1}{\left(1-\varepsilon^{2 / 3} s\right)^{1 / 2}} d s\right) \\
& \lesssim \frac{\exp \left(-\frac{\varepsilon^{-2 / 3}}{4}\right)}{\left(1-\varepsilon^{2 / 3} y\right)^{1 / 2}},
\end{aligned}
$$

where we have also used (3.36). This completes the proof of (3.22) and the proof of Lemma 3.2.

Step 3. Lower bound on $\tilde{\mu}_{n}^{\varepsilon}$ and proof of $(\mathbf{i})_{\mathbf{n}}$. In order to show that $(\mathrm{i})_{\mathrm{n}}$ holds, we next prove the converse inequality

$$
\mu_{n} \leqslant \tilde{\mu}_{n}^{\varepsilon}+\mathcal{O}\left(\varepsilon^{2 / 3-\delta}\right)
$$

which will be deduced from (3.3) and

$$
R\left(\tilde{v}_{n}^{\varepsilon}\right)=\tilde{\mu}_{n}^{\varepsilon}+\mathcal{O}\left(\varepsilon^{2 / 3-\delta}\right), \quad \text { where } \quad \tilde{v}_{n}^{\varepsilon}=\chi_{\varepsilon} \tilde{u}_{n}^{\varepsilon}-\sum_{k=1}^{n-1}\left\langle\chi_{\varepsilon} \tilde{u}_{n}^{\varepsilon}, u_{k}\right\rangle u_{k},
$$


In order to prove (3.39), we proceed similarly as for the proof of (3.5). First, since (iii) $)_{\mathrm{k}}$ is assumed to be satisfied for $k \leqslant n-1$,

$$
\begin{aligned}
R\left(\tilde{v}_{n}^{\varepsilon}\right) & =\frac{Q\left(\chi_{\varepsilon} \tilde{u}_{n}^{\varepsilon}, \chi_{\varepsilon} \tilde{u}_{n}^{\varepsilon}\right)-\sum_{k=1}^{n-1} \mu_{k}\left\langle\chi_{\varepsilon} \tilde{u}_{n}^{\varepsilon}, u_{k}\right\rangle^{2}}{\left\|\chi_{\varepsilon} \tilde{u}_{n}^{\varepsilon}\right\|^{2}-\sum_{k=1}^{n-1}\left\langle\chi_{\varepsilon} \tilde{u}_{n}^{\varepsilon}, u_{k}\right\rangle^{2}} \\
& =\frac{Q\left(\chi_{\varepsilon} \tilde{u}_{n}^{\varepsilon}, \chi_{\varepsilon} \tilde{u}_{n}^{\varepsilon}\right)-\mu_{n-1}\left\langle\chi_{\varepsilon} \tilde{u}_{n}^{\varepsilon}, u_{n-1}\right\rangle^{2}+\mathcal{O}\left(\varepsilon^{2 / 3-\delta}\right)}{\left\|\chi_{\varepsilon} \tilde{u}_{n}^{\varepsilon}\right\|^{2}-\left\langle\chi_{\varepsilon} \tilde{u}_{n}^{\varepsilon}, u_{n-1}\right\rangle^{2}+\mathcal{O}\left(\varepsilon^{2 / 3-\delta}\right)} .
\end{aligned}
$$

Then, thanks to Lemma 3.2 and the normalization of $\tilde{u}_{n}^{\varepsilon}$,

$$
\begin{aligned}
\left\|\chi_{\varepsilon} \tilde{u}_{n}^{\varepsilon}\right\|^{2} & =\int_{-\frac{\varepsilon^{-2 / 3}}{2}}^{\frac{\varepsilon^{-2 / 3}}{2}} \chi_{\varepsilon}^{2}\left|\tilde{u}_{n}^{\varepsilon}\right|^{2} d y \\
& =\left(1+\mathcal{O}\left(\varepsilon^{2 / 3-\delta}\right)\right) \int_{-\varepsilon^{-\delta}}^{\varepsilon^{-\delta}} \frac{\left|\tilde{u}_{n}^{\varepsilon}\right|^{2}}{\left(1-\varepsilon^{2 / 3} y\right)^{1 / 2}} d y+\int_{\varepsilon^{-\delta} \leqslant|y| \leqslant \frac{\varepsilon^{-2 / 3}}{2}} \chi_{\varepsilon}^{2}\left|\tilde{u}_{n}^{\varepsilon}\right|^{2} d y \\
& =\left(1+\mathcal{O}\left(\varepsilon^{2 / 3-\delta}\right)\right) \int_{J_{\varepsilon}} \frac{\left|\tilde{u}_{n}^{\varepsilon}\right|^{2}}{\left(1-\varepsilon^{2 / 3} y\right)^{1 / 2}} d y+\mathcal{O}\left(\varepsilon^{2 / 3}\right) \\
& =1+\mathcal{O}\left(\varepsilon^{2 / 3-\delta}\right) .
\end{aligned}
$$

Similarly, using Lemma 3.2 and (3.18) and proceeding as in (3.15), we get

$$
\begin{aligned}
Q\left(\chi_{\varepsilon} \tilde{u}_{n}^{\varepsilon}, \chi_{\varepsilon} \tilde{u}_{n}^{\varepsilon}\right)= & \int_{-\infty}^{+\infty}\left(4\left|\partial_{y}\left(\chi_{\varepsilon} \tilde{u}_{n}^{\varepsilon}\right)\right|^{2}+W_{0}\left|\chi_{\varepsilon} \tilde{u}_{n}^{\varepsilon}\right|^{2}\right) d y \\
= & 4 \int_{-\infty}^{+\infty} \chi_{\varepsilon}^{\prime 2}\left|\tilde{u}_{n}^{\varepsilon}\right|^{2} d y+8 \int_{-\infty}^{+\infty} \chi_{\varepsilon}^{\prime} \chi_{\varepsilon}\left(\tilde{u}_{n}^{\varepsilon}\right)^{\prime} \tilde{u}_{n}^{\varepsilon} d y \\
& +4\left(1+\mathcal{O}\left(\varepsilon^{2 / 3-\delta}\right)\right) \int_{-\varepsilon^{-\delta}}^{\varepsilon^{-\delta}}\left(1-\varepsilon^{2 / 3} y\right)^{1 / 2}\left|\left(\tilde{u}_{n}^{\varepsilon}\right)^{\prime}\right|^{2} d y \\
& +4 \int_{\varepsilon^{-\delta} \leqslant|y| \leqslant \frac{\varepsilon^{-2 / 3}}{2}} \chi_{\varepsilon}^{2}\left|\left(\tilde{u}_{n}^{\varepsilon}\right)^{\prime}\right|^{2} d y \\
& +\int_{-\varepsilon^{-\delta / 2}}^{\varepsilon^{-\delta / 2}} W_{0}\left|\tilde{u}_{n}^{\varepsilon}\right|^{2} d y+\int_{\varepsilon^{-\delta / 2} \leqslant|y| \leqslant \frac{\varepsilon^{-2 / 3}}{2}} W_{0}\left|\chi_{\varepsilon} \tilde{u}_{n}^{\varepsilon}\right|^{2} d y \\
= & 4\left(1+\mathcal{O}\left(\varepsilon^{2 / 3-\delta}\right)\right) \int_{J_{\varepsilon}}\left(1-\varepsilon^{2 / 3} y\right)^{1 / 2}\left|\left(\tilde{u}_{n}^{\varepsilon}\right)^{\prime}\right|^{2} d y \\
& +\int_{-\varepsilon^{-\delta / 2}}^{\varepsilon^{-\delta / 2}} \frac{W_{\varepsilon}\left|\tilde{u}_{n}^{\varepsilon}\right|^{2}}{\left(1-\varepsilon^{2 / 3} y\right)^{1 / 2}} d y+\mathcal{O}\left(\varepsilon^{2 / 3-\delta}\right) \\
= & Q^{\varepsilon}\left(\tilde{u}_{n}^{\varepsilon}, \tilde{u}_{n}^{\varepsilon}\right)+\mathcal{O}\left(\varepsilon^{2 / 3-\delta}\right)=\tilde{\mu}_{n}^{\varepsilon}+\mathcal{O}\left(\varepsilon^{2 / 3-\delta}\right) .
\end{aligned}
$$

In order to deduce (3.39) from (3.40), it remains to estimate the scalar product $\left\langle\chi_{\varepsilon} \tilde{u}_{n}^{\varepsilon}, u_{n-1}\right\rangle$. Notice that in the case when $n=1$, this term does not exists, and there is then nothing to do. From (iv) $)_{\mathrm{n}-1}$, there exists $c_{n-1} \in \mathbb{R}$ such that

$$
\left\|\chi_{\varepsilon} \tilde{u}_{n-1}^{\varepsilon}-c_{n-1} u_{n-1}\right\| \lesssim \varepsilon^{1 / 3-\delta / 2} .
$$


Then, by triangular inequality, and thanks to (3.41) for $n$ replaced by $n-1$,

$$
|| c_{n-1}|-1| \leqslant\left\|c_{n-1} u_{n-1}-\chi_{\varepsilon} \tilde{u}_{n-1}^{\varepsilon}\right\|+||\left|\chi_{\varepsilon} \tilde{u}_{n-1}^{\varepsilon} \|-1\right| \lesssim \varepsilon^{1 / 3-\delta / 2}
$$

whereas

$$
\begin{aligned}
& \left|c_{n-1}\right|\left|\left\langle\chi_{\varepsilon} \tilde{u}_{n}^{\varepsilon}, u_{n-1}\right\rangle\right| \\
& \quad \leqslant\left|\left\langle\chi_{\varepsilon} \tilde{u}_{n}^{\varepsilon}, c_{n-1} u_{n-1}-\chi_{\varepsilon} \tilde{u}_{n-1}^{\varepsilon}\right\rangle\right|+\left|\left\langle\left(\chi_{\varepsilon}^{2}-\frac{1}{\left(1-\varepsilon^{2 / 3} y\right)^{1 / 2}}\right) \tilde{u}_{n}^{\varepsilon}, \tilde{u}_{n-1}^{\varepsilon}\right\rangle\right|+\left|\left\langle\tilde{u}_{n}^{\varepsilon}, \tilde{u}_{n-1}^{\varepsilon}\right\rangle_{\varepsilon}\right| \\
& \quad \lesssim \varepsilon^{1 / 3-\delta / 2},
\end{aligned}
$$

where the first term in the right hand side of (3.45) has been estimated thanks to the CauchySchwarz inequality, (3.43) and (3.41). The second term has been estimated thanks to Lemma 3.2 for $\tilde{u}_{n}^{\varepsilon}$ and for $\tilde{u}_{n-1}^{\varepsilon}$, and the last one is equal to 0 . We deduce from (3.44) and (3.45) that

$$
\left\langle\chi_{\varepsilon} \tilde{u}_{n}^{\varepsilon}, u_{n-1}\right\rangle=\mathcal{O}\left(\varepsilon^{1 / 3-\delta / 2}\right) .
$$

Then, (3.39) and (3.38) follow from (3.40), (3.41), (3.42) and (3.46). Property (i) $)_{\mathrm{n}}$ is a direct consequence of (3.38) and (3.6).

Step 4. Proof of (ii) $\mathbf{n}$ and (iv) $)_{\mathbf{n}}$. From the definition of $\tilde{v}_{n}^{\varepsilon}$ in (3.39), it is clear that

$$
\tilde{v}_{n}^{\varepsilon} \in \operatorname{Span}\left(u_{1}, \cdots, u_{n-1}\right)^{\perp} .
$$

Thus, $\tilde{v}_{n}^{\varepsilon}$ can be decomposed as

$$
\tilde{v}_{n}^{\varepsilon}=c_{n}^{\varepsilon} u_{n}+w_{n}^{\varepsilon}, \quad \text { where } \quad c_{n}^{\varepsilon} \in \mathbb{R} \text { and } w_{n}^{\varepsilon} \in \operatorname{Span}\left(u_{1}, \cdots, u_{n}\right)^{\perp} .
$$

From (3.39) and (i) $)_{\mathrm{n}}$, we have

$$
\mu_{n}+\mathcal{O}\left(\varepsilon^{2 / 3-\delta}\right)=\tilde{\mu}_{n}^{\varepsilon}+\mathcal{O}\left(\varepsilon^{2 / 3-\delta}\right)=R\left(\tilde{v}_{n}^{\varepsilon}\right)=\frac{\left(c_{n}^{\varepsilon}\right)^{2} \mu_{n}+Q\left(w_{n}^{\varepsilon}, w_{n}^{\varepsilon}\right)}{\left(c_{n}^{\varepsilon}\right)^{2}+\left\|w_{n}^{\varepsilon}\right\|^{2}} \geqslant \frac{\left(c_{n}^{\varepsilon}\right)^{2} \mu_{n}+\left\|w_{n}^{\varepsilon}\right\|^{2} \mu_{n+1}}{\left(c_{n}^{\varepsilon}\right)^{2}+\left\|w_{n}^{\varepsilon}\right\|^{2}} .
$$

It follows that

$$
\left(\mu_{n+1}-\mu_{n}\right)\left\|w_{n}^{\varepsilon}\right\|^{2} \lesssim \varepsilon^{2 / 3-\delta}\left\|\tilde{v}_{n}^{\varepsilon}\right\|^{2} .
$$

Thanks to the definition of $\tilde{v}_{n}^{\varepsilon}$ in (3.39), property (iii) k $_{\mathrm{k}}$ for $k \leqslant n-1$ as well as (3.46),

$$
\left\|\tilde{v}_{n}^{\varepsilon}-\chi_{\varepsilon} \tilde{u}_{n}^{\varepsilon}\right\| \lesssim \varepsilon^{1 / 3-\delta / 2} .
$$

On the other side, (3.41) ensures that $\left\|\chi_{\varepsilon} \tilde{u}_{n}^{\varepsilon}\right\| \rightarrow 1$ as $\varepsilon \rightarrow 0$. As a result, $\left\|\tilde{v}_{n}^{\varepsilon}\right\| \rightarrow 1$ as $\varepsilon \rightarrow 0$, and (3.48) implies

$$
\left\|w_{n}^{\varepsilon}\right\| \lesssim \varepsilon^{1 / 3-\delta / 2}
$$

Moreover, from Lemmas 3.1 and 3.2, we infer that for any $k \geqslant 1$,

$$
\begin{aligned}
\left\langle\chi_{\varepsilon} \tilde{u}_{n}^{\varepsilon}, u_{k}\right\rangle & =\int_{-\varepsilon^{-\delta}}^{\varepsilon^{-\delta}} \chi_{\varepsilon} \tilde{u}_{n}^{\varepsilon} u_{k} d y+\int_{\varepsilon^{-\delta} \leqslant|y| \leqslant \frac{\varepsilon^{-2 / 3}}{2}} \chi_{\varepsilon} \tilde{u}_{n}^{\varepsilon} u_{k} d y \\
& =\left(1+\mathcal{O}\left(\varepsilon^{2 / 3-\delta}\right)\right) \int_{J_{\varepsilon}} \frac{\chi_{\varepsilon} u_{k} \tilde{u}_{n}^{\varepsilon}}{\left(1-\varepsilon^{2 / 3} y\right)^{1 / 2}} d y+\mathcal{O}\left(\varepsilon^{2 / 3}\right) .
\end{aligned}
$$


¿From (3.50) and (3.51) we deduce in particular that for every $k \geqslant n+1$,

$$
\mathcal{O}\left(\varepsilon^{2 / 3}\right)+\left\langle\chi_{\varepsilon} u_{k}, \tilde{u}_{n}^{\varepsilon}\right\rangle_{\varepsilon}\left(1+\mathcal{O}\left(\varepsilon^{2 / 3-\delta}\right)\right)=\left\langle\chi_{\varepsilon} \tilde{u}_{n}^{\varepsilon}, u_{k}\right\rangle=\left\langle\tilde{v}_{n}^{\varepsilon}, u_{k}\right\rangle=\left\langle w_{n}^{\varepsilon}, u_{k}\right\rangle=\mathcal{O}\left(\varepsilon^{1 / 3-\delta / 2}\right),
$$

which proves (ii) $)_{\mathrm{n}}$. Then, (iv) $)_{\mathrm{n}}$ is a consequence of the triangular inequality, (3.49) and (3.50):

$$
\left\|\chi_{\varepsilon} \tilde{u}_{n}^{\varepsilon}-c_{n}^{\varepsilon} u_{n}\right\| \leqslant\left\|\chi_{\varepsilon} \tilde{u}_{n}^{\varepsilon}-\tilde{v}_{n}^{\varepsilon}\right\|+\left\|w_{n}^{\varepsilon}\right\| \lesssim \varepsilon^{1 / 3-\delta / 2} .
$$

Step 5. Proof of (iii) $)_{\mathbf{n}}$ and $(\mathbf{v})_{\mathbf{n}}$. Like in (3.47), we decompose $v_{n-1}^{\varepsilon}$ as

$$
v_{n-1}^{\varepsilon}=\tilde{c}_{n-1}^{\varepsilon} \tilde{u}_{n-1}^{\varepsilon}+\tilde{w}_{n-1}^{\varepsilon}, \quad \text { where } \quad \tilde{c}_{n-1}^{\varepsilon} \in \mathbb{R} \text { and } \tilde{w}_{n-1}^{\varepsilon} \in \operatorname{Span}\left(\tilde{u}_{1}^{\varepsilon}, \cdots, \tilde{u}_{n-1}^{\varepsilon}\right)^{\perp_{\varepsilon}} .
$$

¿From (3.5) for $n$ replaced by $n-1$ and (i) $)_{n-1}$, we have

$$
\begin{aligned}
\tilde{\mu}_{n-1}^{\varepsilon}+\mathcal{O}\left(\varepsilon^{2 / 3-\delta}\right)=\mu_{n-1}+\mathcal{O}\left(\varepsilon^{2 / 3-\delta}\right)=R^{\varepsilon}\left(v_{n-1}^{\varepsilon}\right) & =\frac{\left(\tilde{c}_{n-1}^{\varepsilon}\right)^{2} \tilde{\mu}_{n-1}^{\varepsilon}+Q^{\varepsilon}\left(\tilde{w}_{n-1}^{\varepsilon}, \tilde{w}_{n-1}^{\varepsilon}\right)}{\left(\tilde{c}_{n-1}^{\varepsilon}\right)^{2}+\left\|\tilde{w}_{n-1}^{\varepsilon}\right\|_{\varepsilon}^{2}} \\
& \geqslant \frac{\left(\tilde{c}_{n-1}^{\varepsilon}\right)^{2} \tilde{\mu}_{n-1}^{\varepsilon}+\left\|\tilde{w}_{n-1}^{\varepsilon}\right\|_{\varepsilon}^{2} \tilde{\mu}_{n}^{\varepsilon}}{\left(\tilde{c}_{n-1}^{\varepsilon}\right)^{2}+\left\|\tilde{w}_{n-1}^{\varepsilon}\right\|_{\varepsilon}^{2}} .
\end{aligned}
$$

Using $(\mathrm{i})_{\mathrm{n}}$ and $(\mathrm{i})_{\mathrm{n}-1}$, it follows that

$$
\left(\mu_{n}-\mu_{n-1}+\mathcal{O}\left(\varepsilon^{2 / 3-\delta}\right)\right)\left\|\tilde{w}_{n-1}^{\varepsilon}\right\|_{\varepsilon}^{2}=\left(\tilde{\mu}_{n}^{\varepsilon}-\tilde{\mu}_{n-1}^{\varepsilon}\right)\left\|\tilde{w}_{n-1}^{\varepsilon}\right\|_{\varepsilon}^{2} \lesssim \varepsilon^{2 / 3-\delta}\left\|v_{n-1}^{\varepsilon}\right\|_{\varepsilon}^{2} .
$$

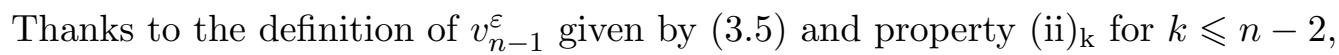

$$
\left\|v_{n-1}^{\varepsilon}-\chi_{\varepsilon} u_{n-1}\right\|_{\varepsilon} \lesssim \varepsilon^{1 / 3-\delta / 2} .
$$

Thanks to (3.14) for $n$ replaced by $n-1,\left\|\chi_{\varepsilon} u_{n-1}\right\|_{\varepsilon} \rightarrow 1$ as $\varepsilon \rightarrow 0$, thus $\left\|v_{n-1}^{\varepsilon}\right\|_{\varepsilon} \rightarrow 1$ as $\varepsilon \rightarrow 0$. As a result, we deduce from (3.53) that

$$
\left\|\tilde{w}_{n-1}^{\varepsilon}\right\|_{\varepsilon} \lesssim \varepsilon^{1 / 3-\delta / 2} .
$$

Then, for every $k \geqslant n$, we get

$$
\left\langle\chi_{\varepsilon} \tilde{u}_{k}^{\varepsilon}, u_{n-1}\right\rangle\left(1+\mathcal{O}\left(\varepsilon^{2 / 3-\delta}\right)\right)=\left\langle\chi_{\varepsilon} u_{n-1}, \tilde{u}_{k}^{\varepsilon}\right\rangle_{\varepsilon}=\left\langle v_{n-1}^{\varepsilon}, \tilde{u}_{k}^{\varepsilon}\right\rangle_{\varepsilon}=\left\langle\tilde{w}_{n-1}^{\varepsilon}, \tilde{u}_{k}^{\varepsilon}\right\rangle_{\varepsilon}=\mathcal{O}\left(\varepsilon^{1 / 3-\delta / 2}\right),
$$

using similar arguments as in the derivation of (3.52). Moreover,

$$
\begin{aligned}
\left\langle\chi_{\varepsilon} u_{n-1}, \tilde{u}_{k}^{\varepsilon}\right\rangle_{\varepsilon}= & \left(1+\mathcal{O}\left(\varepsilon^{2 / 3-\delta}\right)\right)\left(\left\langle\chi_{\varepsilon} \tilde{u}_{k}^{\varepsilon}, u_{n-1}\right\rangle-\int_{\varepsilon^{-\delta} \leqslant|y| \leqslant \frac{\varepsilon^{-2 / 3}}{2}} \chi_{\varepsilon} u_{n-1} \tilde{u}_{k}^{\varepsilon} d y\right) \\
& +\int_{\varepsilon^{-\delta} \leqslant|y| \leqslant \frac{\varepsilon^{-2 / 3}}{2}} \frac{\chi_{\varepsilon} u_{n-1} \tilde{u}_{k}^{\varepsilon}}{\left(1-\varepsilon^{2 / 3} y\right)^{1 / 2}} d y \\
= & \left(1+\mathcal{O}\left(\varepsilon^{2 / 3-\delta}\right)\right)\left\langle\chi_{\varepsilon} \tilde{u}_{k}^{\varepsilon}, u_{n-1}\right\rangle+\mathcal{O}\left(\varepsilon^{2 / 3}\right),
\end{aligned}
$$

where the two integrals in the right hand side of (3.57) have been estimated thanks to the CauchySchwarz inequality, Lemma 3.1 and the normalization condition $\left\|\tilde{u}_{k}^{\varepsilon}\right\|_{\varepsilon}=1$. The combination of (3.56) and (3.57) completes the proof of $(\mathrm{iii})_{\mathrm{n}}$. Then, $(\mathrm{v})_{\mathrm{n}}$ follows from the triangular inequality, (3.54) and (3.55):

$$
\left\|\chi_{\varepsilon} u_{n-1}-\tilde{c}_{n-1}^{\varepsilon} \tilde{u}_{n-1}^{\varepsilon}\right\|_{\varepsilon} \leqslant\left\|\chi_{\varepsilon} u_{n-1}-v_{n-1}^{\varepsilon}\right\|_{\varepsilon}+\left\|\tilde{w}_{n-1}^{\varepsilon}\right\|_{\varepsilon} \lesssim \varepsilon^{1 / 3-\delta / 2} .
$$

It completes the proof of $\left(G_{n}\right)$, and therefore the proof of Theorem 2 . 


\section{Semi-classical limit for eigenvalues of $L_{+}^{\varepsilon}$}

We list here formal results of the semi-classical theory that describe the distribution of eigenvalues of $L_{+}^{\varepsilon}$. We will show that the standard Bohr-Sommerfeld quantization rule does not give the correct asymptotic behavior of the eigenvalues of $L_{+}^{\varepsilon}$ as $\varepsilon \rightarrow 0$ because the potential $V_{\varepsilon}(x)$ depends on $\varepsilon$. Nevertheless, the Bohr-Sommerfeld quantization rule gives the correct scaling $\mathcal{O}\left(\varepsilon^{2 / 3}\right)$ in agreement with the asymptotic limit (3.2) in Theorem 2.

Eigenvalue problem for operator $L_{+}^{\varepsilon}$ can be rewritten in the form

$$
\left(-\partial_{x}^{2}+\varepsilon^{-2} V_{\varepsilon}(x)\right) u(x)=\varepsilon^{-2} \lambda u(x), \quad x \in \mathbb{R} .
$$

By properties of $\eta_{\varepsilon}$ following from Theorem 1, the potential $V_{\varepsilon}(x)$ has the properties

- $V_{\varepsilon}(x) \in \mathcal{C}^{\infty}(\mathbb{R})$ for any small $\varepsilon>0$,

- $\lim _{\varepsilon \rightarrow 0} V_{\varepsilon}(x)=V_{0}(x)$, where $V_{0} \in \mathcal{C}(\mathbb{R})$ is given by

$$
V_{0}(x)= \begin{cases}2\left(1-x^{2}\right), & |x| \leqslant 1 \\ x^{2}-1, & |x| \geqslant 1\end{cases}
$$

- $V_{\varepsilon}(x)$ takes its absolute minimum at $\pm a_{\varepsilon}$ for any small $\varepsilon \geqslant 0$ and $a_{\varepsilon} \rightarrow 1$ as $\varepsilon \rightarrow 0$,

- $V_{\varepsilon}(x) \rightarrow+\infty$ as $|x| \rightarrow \infty$ for any small $\varepsilon \geqslant 0$.

If $V_{\varepsilon}(x)$ is replaced by $V_{0}(x)$, the eigenvalue problem (4.1) takes a simplified form

$$
\left(-\partial_{x}^{2}+\varepsilon^{-2} V_{0}(x)\right) u(x)=\varepsilon^{-2} \lambda u(x), \quad x \in \mathbb{R},
$$

which describes the eigenvalues of the operator $\tilde{L}_{+}^{\varepsilon}$ mentioned in section 1 . As it is well-known (see a recent review in [BDS]), the eigenvalues of the Schrödinger operator $-\partial_{x}^{2}+\varepsilon^{-2} V(x)$, with a smooth, $\varepsilon$-independent double well potential $V(x)$, are twice degenerate in the semi-classical limit $\varepsilon \rightarrow 0$. Namely, the eigenvalues are grouped by pairs. In each pair, the two eigenvalues are exponentially close one from another as $\varepsilon \rightarrow 0$. The asymptotic distribution of these pairs of eigenvalues is determined by the Bohr-Sommerfeld quantization rule.

Let us try to apply the Bohr-Sommerfeld quantization rule to the eigenvalue problems (4.1) and (4.2) for the operators $L_{+}^{\varepsilon}$ and $\tilde{L}_{+}^{\varepsilon}$, in spite of the fact that this rule was proved rigorously by Fedoryuk [Fed] only for a class of $\varepsilon$-independent, analytic potentials. Since neither (4.1) nor (4.2) satisfies assumptions of the main theorem in [Fed], this application is purely formal. According to the standard Bohr-Sommerfeld rule, the consequent eigenvalues $\lambda_{2 n-1}^{\varepsilon}$ and $\lambda_{2 n}^{\varepsilon}$ of the Schrödinger equation (4.1) with the double-well potential $V_{\varepsilon}(x)$ would be given asymptotically by

$$
\int_{x_{-}^{\varepsilon}(\lambda)}^{x_{+}^{\varepsilon}(\lambda)} \sqrt{\lambda-V_{\varepsilon}(x)} d x \sim \varepsilon \pi\left(n-\frac{1}{2}\right), \quad \text { as } \varepsilon \rightarrow 0, \text { for fixed } n \geqslant 1,
$$

where $x_{ \pm}^{\varepsilon}(\lambda)$ are the roots of $V_{\varepsilon}(x)=\lambda$ on $\mathbb{R}_{+}$, such that $0<x_{-}^{\varepsilon}(\lambda)<1<x_{+}^{\varepsilon}(\lambda)<\infty$. Let us use the scaling

$$
y=\frac{1-x^{2}}{\varepsilon^{2 / 3}}, \quad V_{\varepsilon}(x)=\varepsilon^{2 / 3} W_{\varepsilon}(y), \quad \lambda=\varepsilon^{2 / 3} \mu,
$$


where $W_{\varepsilon}(y)=3 \nu_{\varepsilon}^{2}(y)-y$ and $\mu$ is a new eigenvalue. The Bohr-Sommerfeld rule is rewritten in an equivalent form by

$$
\int_{y_{-}^{\varepsilon}(\mu)}^{y_{+}^{\varepsilon}(\mu)} \frac{\sqrt{\mu-W_{\varepsilon}(y)}}{\sqrt{1-\varepsilon^{2 / 3} y}} d y \sim \pi(2 n-1), \quad \text { as } \quad \varepsilon \rightarrow 0, \text { for fixed } n \geqslant 1,
$$

where $y_{ \pm}^{\varepsilon}(\mu)$ are the roots of $W_{\varepsilon}(y)=\mu$ on $\mathbb{R}$, such that $-\infty<y_{-}^{\varepsilon}(\mu)<0<y_{+}^{\varepsilon}(\mu)<\infty$. Taking the limit $\varepsilon \rightarrow 0$ for a fixed $n \geqslant 1$, we obtain

$$
\int_{y_{-}(\mu)}^{y_{+}(\mu)} \sqrt{\mu-W_{0}(y)} d y \sim \pi(2 n-1), \quad \text { for fixed } n \geqslant 1,
$$

where $W_{0}(y)=3 \nu_{0}^{2}(y)-y$ and $y_{ \pm}(\mu)$ are the roots of $W_{0}(y)=\mu$ on $\mathbb{R}$. The new expression is the Bohr-Sommerfeld quantization rule for the Schrödinger operator $M_{0}=-4 \partial_{y}^{2}+W_{0}$ and it is only valid for large $n \gg 1$. Therefore, the Bohr-Sommerfeld quantization rule (4.3) does not recover the statement of Theorem 2 correctly. Meantime, it still implies that the eigenvalues $\lambda_{2 n-1}^{\varepsilon}$ and $\lambda_{2 n}^{\varepsilon}$ for a fixed $n \geqslant 1$ are scaled as $\mathcal{O}\left(\varepsilon^{2 / 3}\right)$ as $\varepsilon \rightarrow 0$. The discrepancy of the Bohr-Sommerfeld rule is explained by the fact that the smooth potential $V_{\varepsilon}(x)$ in the eigenvalue problem (4.1) depends on $\varepsilon$.

Note that the limit $\varepsilon \rightarrow 0$ can be computed exactly for the simplified eigenvalue problem (4.2) thanks to the scaling transformation (4.4). In this case, the limiting formula (4.5) holds with $W_{0}(y)$ replaced by $2 y$ for $y \geqslant 0$ and $-y$ for $y \leqslant 0$, so that $y_{-}(\mu)=-\mu$ and $y_{+}(\mu)=\mu / 2$. In other words,

$$
\int_{-\mu}^{0} \sqrt{\mu+y} d y+\int_{0}^{\mu / 2} \sqrt{\mu-2 y} d y \sim \pi(2 n-1), \quad \text { for fixed } n \geqslant 1,
$$

and the computations of integrals gives $\mu_{n} \sim(\pi(2 n-1))^{2 / 3}$, in agreement with the behavior $\mathcal{O}\left(n^{2 / 3}\right)$ of eigenvalues of the Schrödinger operator with a linearly growing potential as $|y| \rightarrow \infty$ [Su]. Therefore, the Bohr-Sommerfeld quantization rule suggests that the eigenvalues $\left\{\tilde{\lambda}_{n}^{\varepsilon}\right\}_{n \geqslant 1}$ of the simplified operator $\tilde{L}_{+}^{\varepsilon}$ considered in our previous work [GP] satisfy the asymptotic limit

$$
\lim _{\varepsilon \downarrow 0} \frac{\tilde{\lambda}_{2 n-1}^{\varepsilon}}{\varepsilon^{2 / 3}}=\lim _{\varepsilon \downarrow 0} \frac{\tilde{\lambda}_{2 n}^{\varepsilon}}{\varepsilon^{2 / 3}}=(\pi(2 n-1))^{2 / 3}, \quad \text { for fixed } n \geqslant 1 .
$$

However, the justification of the asymptotic limit (4.6) cannot rely on the work of Fedoryuk [Fed] because the $\varepsilon$-independent potential $V_{0}(x)$ in the simplified eigenvalue problem (4.2) is continuous but not $\mathcal{C}^{1}$ on $\mathbb{R}$.

\section{$5 \quad$ Proof of Proposition 1.1}

For a radial function $u(x)=u(|x|)$ solution to (1.2), (1.2) can be rewritten as

$$
\frac{\varepsilon^{2}}{r^{d-1}} \frac{d}{d r}\left(r^{d-1} u^{\prime}\right)+\left(1-r^{2}-u^{2}\right) u=0, \quad r \geqslant 0, \quad u^{\prime}(0)=0 .
$$

Let $u, v \in L^{2}\left(\mathbb{R}^{d}\right)$ be two radial positive solutions of (5.1). Up to a change of $u$ and $v$, we assume $u(0) \leqslant v(0)$. Let $\rho=u / v$. Then, a straightforward calculation shows that

$$
\varepsilon^{2} \frac{d}{d r}\left(v^{2} r^{d-1} \rho^{\prime}\right)=r^{d-1} v^{4} \rho\left(\rho^{2}-1\right)
$$


If $u(0)=v(0)$, since $u^{\prime}(0)=v^{\prime}(0)=0$, we have $u \equiv v$. Let us assume by contradiction $u(0)<v(0)$, that is, $\rho(0)<1$. Then $r \mapsto v(r)^{2} r^{d-1} \rho^{\prime}(r)$ is strictly decreasing in a neighborhood of 0 , and therefore, since $\rho^{\prime}(0)=0$, we infer $\rho^{\prime}(r)<0$ for $r>0$ sufficiently small. Let

$$
r_{0}=\inf \left\{r>0, \rho^{\prime}(r)=0\right\} .
$$

$\rho$ is decreasing on $\left(0, r_{0}\right)$, and since for every $r, 0<\rho(r)<\rho(0)<1$, we infer that $v^{4} \rho\left(\rho^{2}-1\right)<0$ on that interval. As a result, $r \mapsto v(r)^{2} r^{d-1} \rho^{\prime}(r)$ is decreasing on $\left(0, r_{0}\right)$. Moreover, if $r_{0}$ was finite, we would have $v\left(r_{0}\right)^{2} r_{0}^{d-1} \rho^{\prime}\left(r_{0}\right)<0$, which would be a contradiction with the definition of $r_{0}$. Thus $r_{0}=+\infty$, such that both $\rho$ and $v(r)^{2} r^{d-1} \rho^{\prime}(r)$ are decreasing on $\mathbb{R}_{+}$. We deduce

$$
-1 \leqslant \rho(\infty)-\rho(1)=\int_{1}^{\infty} \rho^{\prime}(r) d r \leqslant v(1)^{2} \rho^{\prime}(1) \int_{1}^{\infty} \frac{1}{v(r)^{2} r^{d-1}} d r<0 .
$$

In particular, the integral

$$
\int_{1}^{\infty} \frac{1}{v(r)^{2} r^{d-1}} d r<+\infty
$$

converges. On the other side, since $v \in L^{2}\left(\mathbb{R}^{d}\right)$,

$$
\int_{0}^{\infty} v(r)^{2} r^{d-1} d r<+\infty
$$

Thanks to the Cauchy-Schwarz inequality, it turns out that

$$
\infty=\int_{1}^{\infty} d r \leqslant\left(\int_{1}^{\infty} v(r)^{2} r^{d-1} d r\right)^{1 / 2}\left(\int_{1}^{\infty} \frac{1}{v(r)^{2} r^{d-1}} d r\right)^{1 / 2}<\infty,
$$

which gives contradiction. Thus, $\rho(0)=1$ and $u \equiv v$.

\section{Proof of Lemma 2.1}

Let $\alpha>1$ be like in the assumption of the lemma, and $A=\left\|x^{\alpha} f\right\|_{L^{\infty}\left(\mathbb{R}_{+}\right)}<\infty$. We first prove (2.11) by contradiction. We proceed as follows. We suppose that (2.11) is not true. Namely, we make the assumption

$$
\varphi(x) \neq \mathcal{O}\left(x^{-(\alpha+1)}\right), \quad \quad x^{\alpha} f \in L^{\infty}\left(A_{+},+\infty\right)
$$

If $\alpha>2$, we prove that $\left(\mathrm{G}_{\alpha}\right)$ implies $\left(\mathrm{G}_{\alpha-2}\right)$, such that after a finite number of steps, $\left(\mathrm{G}_{\alpha}\right)$ implies $\left(\mathrm{G}_{\tilde{\alpha}}\right)$ for some $\tilde{\alpha} \in(0,2]$. On the other side, we show that for $0<\alpha \leqslant 2,\left(\mathrm{G}_{\alpha}\right)$ yields a contradiction.

If (2.11) is not true, then, up to a change of $f$ and $\varphi$ into $-f$ and $-\varphi$, there exists a sequence $\left(x_{n}\right)_{n \geqslant n_{0}}$ (where $n_{0}>A$ ), such that $x_{n} \uparrow \infty, x_{n} \geqslant A_{+}$and

$$
x_{n}^{\alpha} W\left(x_{n}\right) \varphi\left(x_{n}\right)>n \text {. }
$$

Then,

$$
x_{n}^{\alpha} \varphi^{\prime \prime}\left(x_{n}\right)=x_{n}^{\alpha} W\left(x_{n}\right) \varphi\left(x_{n}\right)-x_{n}^{\alpha} f(x) \geqslant x_{n}^{\alpha} W\left(x_{n}\right) \varphi\left(x_{n}\right)-A>n-A .
$$

For $n \geqslant n_{0}>A$, we define

$$
y_{n}=\sup \left\{y>x_{n}, \forall x \in\left(x_{n}, y\right), x^{\alpha} W(x) \varphi(x)-A>(n-A) / 2\right\} .
$$


By continuity of $W$ and $\varphi$, for every $n \geqslant n_{0}$, either $y_{n}=+\infty$ or

$$
\varphi\left(y_{n}\right)=\frac{n+A}{2 y_{n}^{\alpha} W\left(y_{n}\right)} .
$$

We distinguish the two following cases:

A) There exists $n_{1} \geqslant n_{0}$ such that $y_{n_{1}}=+\infty$

B) For every $n \geqslant n_{0}, y_{n}<+\infty$.

In case B), extracting a subsequence of $\left(x_{n}\right)_{n \geqslant n_{0}}$ if necessary, one can assume that

$$
x_{n_{0}}<y_{n_{0}}<x_{n_{0}+1}<y_{n_{0}+1}<x_{n_{0}+2}<\cdots
$$

For $n \geqslant n_{0}+1$, we define

$$
\tilde{x}_{n}=\inf \left\{y<x_{n}, \forall x \in\left(y, x_{n}\right), x^{\alpha} W(x) \varphi(x)-A>3(n-A) / 4\right\} .
$$

Since $y_{n-1}<x_{n}$ and

$$
y_{n-1}^{\alpha} W\left(y_{n-1}\right) \varphi\left(y_{n-1}\right)-A=(n-1-A) / 2<3(n-A) / 4,
$$

we deduce $\tilde{x}_{n}>y_{n-1}>-\infty$. Moreover, by continuity, $\varphi\left(\tilde{x}_{n}\right)=(3 n+A) /\left(4 \tilde{x}_{n}^{\alpha} W\left(\tilde{x}_{n}\right)\right)$, and $\varphi(x)>(3 n+A) /\left(4 x^{\alpha} W(x)\right)$ for $x>\tilde{x}_{n}, x$ close to $\tilde{x}_{n}$. Therefore

$$
\begin{aligned}
\varphi^{\prime}\left(\tilde{x}_{n}\right) \geqslant \frac{3 n+A}{4} \frac{d}{d x}\left(\frac{1}{x^{\alpha} W(x)}\right)_{\mid x=\tilde{x}_{n}} & \geqslant-\frac{3 n+A}{4}\left(\alpha+\frac{\left\|W^{\prime}\right\|_{L^{\infty}}}{C_{+}}\right) \frac{1}{\tilde{x}_{n}^{\alpha+1} W\left(\tilde{x}_{n}\right)} \\
& \geqslant-C_{1} n \frac{1}{\tilde{x}_{n}^{\alpha+1} W\left(\tilde{x}_{n}\right)},
\end{aligned}
$$

for some $C_{1}>0$. By definition of $y_{n}$ and $\tilde{x}_{n}$, for every $x \in\left(\tilde{x}_{n}, y_{n}\right)$,

$$
x^{\alpha} \varphi^{\prime \prime}(x) \geqslant \frac{n-A}{2} \text {. }
$$

Thus,

$$
\begin{aligned}
\varphi^{\prime}(x) & \geqslant \varphi^{\prime}\left(\tilde{x}_{n}\right)+\frac{n-A}{2} \int_{\tilde{x}_{n}}^{x} \frac{1}{y^{\alpha}} d y \\
& \geqslant-C_{1} n \frac{1}{\tilde{x}_{n}^{\alpha+1} W\left(\tilde{x}_{n}\right)}+\frac{n-A}{2} \int_{\tilde{x}_{n}}^{x} \frac{1}{y^{\alpha}} d y=: G_{n}(x) .
\end{aligned}
$$

Notice that $G_{n}\left(\tilde{x}_{n}\right)<0$, whereas

$$
G_{n}(+\infty)= \begin{cases}+\infty & \text { if } \alpha \leqslant 1 \\ g_{n} & \text { if } \alpha>1\end{cases}
$$

where for $\alpha>1$,

$$
g_{n} \sim \frac{n-A}{2(\alpha-1) \tilde{x}_{n}^{\alpha-1}}>0 \text { as } n \rightarrow+\infty .
$$

As a result, for $n$ sufficiently large, since $G_{n}$ is increasing on $\left(\tilde{x}_{n},+\infty\right), G_{n}$ vanishes exactly once on that interval. Moreover, this unique zero $z_{n}$ of $G_{n}$ is defined by

$$
\int_{\tilde{x}_{n}}^{z_{n}} \frac{1}{y^{\alpha}} d y=\frac{2 C_{1} n}{n-A} \frac{1}{\tilde{x}_{n}^{\alpha+1} W\left(\tilde{x}_{n}\right)},
$$


thus

$$
z_{n}=\tilde{x}_{n}+\mathcal{O}\left(\frac{1}{\tilde{x}_{n}^{2}}\right)
$$

By integration of $(6.2)$, we infer that for $x \in\left(\tilde{x}_{n}, y_{n}\right)$,

$$
\begin{aligned}
\varphi(x) & \geqslant \varphi\left(\tilde{x}_{n}\right)+\int_{\tilde{x}_{n}}^{x} G_{n}(y) d y \\
& \geqslant \varphi\left(\tilde{x}_{n}\right)+\int_{\tilde{x}_{n}}^{z_{n}} G_{n}(y) d y \\
& \geqslant \varphi\left(\tilde{x}_{n}\right)-\frac{C_{1} n}{\tilde{x}_{n}^{\alpha+1} W\left(\tilde{x}_{n}\right)}\left(z_{n}-\tilde{x}_{n}\right) \\
& \geqslant \frac{3 n+A}{4} \frac{1}{\tilde{x}_{n}^{\alpha} W\left(\tilde{x}_{n}\right)}-\frac{C_{2} n}{\tilde{x}_{n}^{\alpha+3} W\left(\tilde{x}_{n}\right)},
\end{aligned}
$$

for some constant $C_{2}>0$. Therefore, for $n$ large enough, for every $x \in\left(\tilde{x}_{n}, y_{n}\right)$, since $W$ is increasing on $\left(A_{+},+\infty\right)$,

$$
\varphi(x) \geqslant \frac{5 n}{8} \frac{1}{\tilde{x}_{n}^{\alpha} W\left(\tilde{x}_{n}\right)} \geqslant \frac{5 n}{8} \frac{1}{x^{\alpha} W(x)}
$$

For $n$ sufficiently large, $5 n / 8>(n+A) / 2$, and it provides a contradiction with (6.1), which means that case B) can not happen. In case A), for every $x \geqslant x_{n_{1}}$,

$$
x^{\alpha} \varphi^{\prime \prime}(x) \geqslant\left(n_{1}-A\right) / 2>0 .
$$

Therefore $\varphi^{\prime}(x) \uparrow 0$ as $x \uparrow \infty$, otherwise $\varphi$ would not be in $L^{2}(\mathbb{R})$. Thus, for every $x \geqslant x_{n_{1}}$, $\varphi^{\prime}(x) \leqslant 0$, and therefore $\varphi(x) \downarrow 0$ as $x \uparrow \infty$. If $0<\alpha \leqslant 1$, (6.3) provides a contradiction with the fact that $\varphi^{\prime}(x) \rightarrow 0$ as $x \rightarrow \infty$. If $\alpha>1$, integration of (6.3) between $x$ and $+\infty$ yields

$$
-\varphi^{\prime}(x) \geqslant \frac{n_{1}-A}{2(\alpha-1)} x^{1-\alpha} .
$$

This is a contradiction with $\varphi(x) \rightarrow 0$, if $1<\alpha \leqslant 2$. Finally, if $\alpha>2$, by integration of (6.4),

$$
\varphi(x) \geqslant \frac{n_{1}-A}{2(\alpha-1)} \int_{x}^{+\infty} y^{1-\alpha} d y=\frac{n_{1}-A}{2(\alpha-1)(\alpha-2)} x^{2-\alpha} .
$$

Thus,

$$
\varphi(x) \underset{x \rightarrow \infty}{\neq} \mathcal{O}\left(x^{-(\alpha-2)-1}\right) .
$$

Since the assumption $x^{\alpha} f \in L^{\infty}\left(A_{+},+\infty\right)$ implies $x^{\alpha-2} f \in L^{\infty}\left(A_{+},+\infty\right)$, we have proved that $\left(\mathrm{G}_{\alpha}\right)$ implies $\left(\mathrm{G}_{\tilde{\alpha}}\right)$ if $\alpha>2$. The proof of (2.11) is completed by induction. Then, since $\varphi^{\prime \prime}=$ $W \varphi-f$, we deduce

$$
\varphi^{\prime \prime}(x)=\mathcal{O}\left(x^{-\alpha}\right)
$$

We next prove that

$$
\varphi^{\prime}(x)= \begin{cases}\mathcal{O}\left(x^{-(\alpha-1)}\right) & \text { if } \alpha>1 \\ o(1) & \text { if } 0<\alpha \leqslant 1\end{cases}
$$


By integration of (6.5), if $\alpha>1, \varphi^{\prime}(x)$ has a limit as $x \rightarrow+\infty$. This limit can only be 0 , because $\varphi \in L^{2}$. (6.6) is then obtained by integration of (6.5) between $x$ an $+\infty$. If $\alpha \leqslant 1,(6.6)$ is a consequence of the fact that $\varphi(x) \rightarrow 0$ and $\varphi^{\prime \prime}(x) \rightarrow 0$ as $x \rightarrow+\infty$.

Let $\chi \in \mathcal{C}^{\infty}(\mathbb{R})$ be such that

$$
\chi(x)= \begin{cases}0 & \text { if } x \leqslant 1 \\ 1 & \text { if } x \geqslant 2\end{cases}
$$

For $m \in \mathbb{N}$, let $\varphi_{m}, f_{m} \in \mathcal{C}^{\infty}(\mathbb{R})$ be the functions defined by

$$
\varphi_{m}(x)=\chi(x) x^{-(\alpha+\gamma m+1)}
$$

and

$$
f_{m}(x)=-\varphi_{m}^{\prime \prime}(x)+W(x) \varphi_{m}(x)
$$

¿From now on, we assume that $f$ and $W$ have asymptotic series (2.12) as $x \rightarrow+\infty$, so that

$$
\begin{aligned}
f_{m}(x) \underset{x \rightarrow+\infty}{\underset{x \rightarrow}{\approx}} x^{-(\alpha+\gamma m)} \sum_{k=0}^{+\infty} v_{k} x^{-\gamma k}+(\alpha+\gamma m+1)(\alpha+\gamma m+2) x^{-(\alpha+\gamma(m+3 / \gamma))} . \\
\underset{x \rightarrow+\infty}{\approx} x^{-(\alpha+\gamma m)} \sum_{k=0}^{+\infty} \tilde{v}_{k} x^{-\gamma k}
\end{aligned}
$$

where $\tilde{v}_{k}=v_{k}$ if $k \neq 3 / \gamma$ and $\tilde{v}_{3 / \gamma}=v_{3 / \gamma}+(\alpha+\gamma m+1)(\alpha+\gamma m+2)$. Notice also that the assumption $W(x) \geqslant C_{+} x$ implies $v_{0} \geqslant C_{+}>0$. As a result, there exists coefficients $\left(\tilde{c}_{m}\right)_{m \in \mathbb{N}}$ such that for every $M \geqslant 0$,

$$
f(x)=\sum_{m=0}^{M} \tilde{c}_{m} f_{m}(x)+g_{M}(x),
$$

where $g_{M}(x)=\mathcal{O}\left(x^{-\alpha-\gamma(M+1)}\right)$ as $x \rightarrow+\infty$. Then,

$$
\varphi(x)=\left(-\partial_{x}^{2}+W\right)^{-1} f(x)=\sum_{m=0}^{M} \tilde{c}_{m} \varphi_{m}(x)+\psi_{M}(x),
$$

where $\psi_{M}=\left(-\partial_{x}^{2}+W\right)^{-1} g_{M}$. Thanks to (2.11), (6.6) and (6.5), for $M$ large enough, $\psi_{M}(x)=$ $\mathcal{O}\left(x^{-\alpha-\gamma(M+1)-1}\right), \psi_{M}^{\prime}(x)=\mathcal{O}\left(x^{-\alpha-\gamma(M+1)+1}\right)$ and $\psi_{M}^{\prime \prime}(x)=\mathcal{O}\left(x^{-\alpha-\gamma(M+1)}\right)$. Since this is true for arbitrarily large values of $M$, then (2.13) and (2.14) follow.

\section{$7 \quad$ Proof of Lemma 2.2}

By Proposition 2.1, we know that $\nu_{0}$ is a strictly increasing function on $\mathbb{R}$, with asymptotics at $\pm \infty$ given by (2.9) and (2.10). Moreover, $\nu_{0}$ has a unique inflection point. From the behaviour of $\nu_{0}(y)$ as $y \rightarrow \pm \infty$, we infer that $W_{0}(y)=3 \nu_{0}(y)^{2}-y \rightarrow+\infty$ as $y \rightarrow \pm \infty$. We are going to prove that the global minimum of $W_{0}$ is actually strictly positive. We argue by contradiction. If it is not the case, we can define

$$
y_{1}=\inf \left\{y>0, \nu_{0}(y)=\sqrt{y / 3}\right\},
$$


where we recall that $W_{0}(y)>0$ if $y \leqslant 0$. By continuity, $\nu_{0}\left(y_{1}\right)=\sqrt{y_{1} / 3}$. We also denote the unique inflection point of $\nu_{0}$ by $y_{0}$. Since $\nu_{0}>0$ solves $(2.5), y_{0}>0$ is the unique solution of the equation $\nu_{0}\left(y_{0}\right)=\sqrt{y_{0}}$, and $\nu_{0}^{\prime \prime}(y)>0$ if $y<y_{0}$, whereas $\nu_{0}^{\prime \prime}(y)<0$ if $y>y_{0}$. Notice that since $\nu_{0}(0)>0$ and $\nu_{0}\left(y_{1}\right)=\sqrt{y_{1} / 3}<\sqrt{y_{1}}$, we have necessarily $0<y_{0}<y_{1}$. Moreover, since $\nu_{0}$ is strictly increasing, we have $\sqrt{y_{0}}=\nu_{0}\left(y_{0}\right)<\nu_{0}\left(y_{1}\right)=\sqrt{y_{1} / 3}$, and therefore $0<3 y_{0}<y_{1}$.

First step: upper bound on $\mathbf{y}_{\mathbf{1}}$. For $y>0$, we introduce the function $z(y)=\nu_{0}(y) / \sqrt{y}$ and rewrite (2.5) in terms of $z(y)$ as

$$
z^{\prime \prime}(y)+\frac{1}{y} z^{\prime}(y)=\frac{y z(y)}{4}\left(z(y)^{2}-1+\frac{1}{y^{3}}\right) .
$$

Since $z(y) \rightarrow+\infty$ as $y \rightarrow 0^{+}$and $z(y) \rightarrow 1$ as $y \rightarrow+\infty$ with $z(y)<1$ for $y$ large enough (because for $y>y_{0}, \nu_{0}^{\prime \prime}(y)<0$ and therefore $\left.\nu_{0}(y)<\sqrt{y}\right)$, we deduce that $z(y)$ admits a global minimum at $y=y_{m}>0$, where

$$
0 \leqslant z^{\prime \prime}\left(y_{m}\right)=\frac{y_{m} z\left(y_{m}\right)}{4}\left(z\left(y_{m}\right)^{2}-1+\frac{1}{y_{m}^{3}}\right)
$$

The assumption of non-positivity of $W_{0}$ implies that $z\left(y_{m}\right) \leqslant 1 / \sqrt{3}$. Thus,

$$
\frac{1}{y_{m}^{3}} \geqslant 1-z\left(y_{m}\right)^{2} \geqslant \frac{2}{3}
$$

As a result, since $\nu_{0}\left(y_{m}\right) \leqslant \sqrt{y_{m} / 3}$

$$
3 y_{0}<y_{1} \leqslant y_{m} \leqslant\left(\frac{3}{2}\right)^{1 / 3}
$$

Second step: upper bound on $\nu_{\mathbf{0}}^{\prime}\left(\mathbf{y}_{\mathbf{0}}\right)$. Since $\nu_{0}$ is increasing on $\mathbb{R}$ and $\nu_{0}(y)^{2}-y>0$ if $y<y_{0}$, we deduce, for every $y<y_{0}$,

$$
\begin{aligned}
\nu_{0}^{\prime}\left(y_{0}\right)-\nu_{0}^{\prime}(y) & =\int_{y}^{y_{0}} \frac{\nu_{0}(t)}{4}\left(\nu_{0}(t)^{2}-t\right) d t \\
& \leqslant \int_{y}^{y_{0}} \frac{\nu_{0}\left(y_{0}\right)}{4}\left(\nu_{0}\left(y_{0}\right)^{2}-t\right) d t=\frac{\sqrt{y_{0}}}{8}\left(y_{0}-y\right)^{2} .
\end{aligned}
$$

By integration, it follows that for $y<y_{0}$,

$$
\begin{aligned}
\nu_{0}(y) & =\sqrt{y_{0}}-\int_{y}^{y_{0}} \nu_{0}^{\prime}(t) d t \\
& \leqslant \sqrt{y_{0}}-\nu_{0}^{\prime}\left(y_{0}\right)\left(y_{0}-y\right)+\frac{\sqrt{y_{0}}}{24}\left(y_{0}-y\right)^{3} .
\end{aligned}
$$

The right hand side reaches its minimum (for $y<y_{0}$ ) at $y=y_{p}$, where $y_{p}<y_{0}$ is defined by $\left(y_{0}-y_{p}\right)^{2}=8 \nu_{0}^{\prime}\left(y_{0}\right) / \sqrt{y_{0}}$, and $(7.3)$ at $y=y_{p}$ yields

$$
\nu_{0}\left(y_{p}\right) \leqslant \sqrt{y_{0}}-\frac{4 \sqrt{2}}{3} \frac{\nu_{0}^{\prime}\left(y_{0}\right)^{3 / 2}}{y_{0}^{1 / 4}} .
$$


Since $\nu_{0}>0$, the right hand side has to be strictly positive. Therefore

$$
\nu_{0}^{\prime}\left(y_{0}\right) \leqslant\left(\frac{9}{32}\right)^{1 / 3} \sqrt{y_{0}} .
$$

Third step: upper bound on $\nu_{\mathbf{0}}^{\prime}\left(\mathbf{y}_{\mathbf{1}}\right)$. On the one side, notice that for $y>y_{0}, \nu_{0}^{\prime \prime}(y)<0$, and therefore $\nu_{0}^{\prime}\left(y_{1}\right) \leqslant \nu_{0}^{\prime}\left(y_{0}\right)$. On the other side, if $y<y_{1}, \nu_{0}(y)^{2}>y / 3$, and $\nu_{0}\left(y_{1}\right)^{2}=y_{1} / 3$, thus

$$
\nu_{0}^{\prime}\left(y_{1}\right) \leqslant\left.\frac{d}{d y} \sqrt{\frac{y}{3}}\right|_{y=y_{1}}=\frac{1}{2 \sqrt{3 y_{1}}} .
$$

As a result, thanks to (7.4) and (7.1)

$$
\nu_{0}^{\prime}\left(y_{1}\right) \leqslant \min \left(\left(\frac{9}{32}\right)^{1 / 3} \frac{\sqrt{y_{1}}}{\sqrt{3}}, \frac{1}{2 \sqrt{3 y_{1}}}\right) .
$$

Fourth step: upper bound on $\nu_{\mathbf{0}}^{\prime}(\mathbf{y})$ for $\mathbf{y}>\mathbf{y}_{\mathbf{1}}$. For $\delta \in(0,2 / 3)$ to be fixed later, we define

$$
y_{2}(\delta)=\sup \left\{y>y_{1}, \forall t \in\left(y_{1}, y\right), \nu_{0}(t)^{2} \leqslant(1-\delta) t\right\}
$$

(notice that $\left.\nu_{0}\left(y_{1}\right)^{2}=y_{1} / 3<(1-\delta) y_{1}\right)$. Then, for every $y \in\left(y_{1}, y_{2}(\delta)\right.$ ),

$$
\begin{aligned}
\nu_{0}^{\prime}(y) & =\nu_{0}^{\prime}\left(y_{1}\right)+\int_{y_{1}}^{y} \frac{\nu_{0}(t)}{4}\left(\nu_{0}(t)^{2}-t\right) d t \\
& \leqslant \nu_{0}^{\prime}\left(y_{1}\right)+\int_{y_{1}}^{y} \frac{\nu_{0}\left(y_{1}\right)}{4}(-\delta t) d t \\
& \leqslant \nu_{0}^{\prime}\left(y_{1}\right)-\frac{\nu_{0}\left(y_{1}\right)}{8} \delta\left(y^{2}-y_{1}^{2}\right) .
\end{aligned}
$$

Fifth step: bound from below on $\mathbf{y}_{\mathbf{2}}(\delta)$. For $\delta \in(0,2 / 3)$, we introduce the function $h_{\delta}$ defined for $y>y_{1}$ by

$$
h_{\delta}(y):=\nu_{0}^{\prime}\left(y_{1}\right)-\frac{\nu_{0}\left(y_{1}\right)}{8} \delta\left(y^{2}-y_{1}^{2}\right)-\frac{\sqrt{1-\delta}}{2 \sqrt{y}} .
$$

¿From (7.5) and since $\delta<2 / 3$, we infer $h_{\delta}\left(y_{1}\right)<0$. Thus, if we define

$$
y_{3}(\delta):=\sup \left\{y>y_{1}, \forall t \in\left(y_{1}, y\right), h_{\delta}(t)<0\right\},
$$

we deduce from (7.6) that for $y \in\left(y_{1}, \min \left(y_{2}(\delta), y_{3}(\delta)\right)\right)$,

$$
\nu_{0}(y)-\sqrt{1-\delta} \sqrt{y}=\nu_{0}\left(y_{1}\right)-\sqrt{1-\delta} \sqrt{y_{1}}+\int_{y_{1}}^{y} h_{\delta}(t) d t<0,
$$

which implies that

$$
y_{3}(\delta) \leqslant y_{2}(\delta)
$$


Sixth step: $\mathbf{y}_{\mathbf{3}}=+\infty$. We shall see next that for an appropriate choice of $\delta, y_{3}(\delta)=$ $+\infty$, which implies that $y_{2}(\delta)=+\infty$ thanks to (7.8). This provides a contradiction with the assumption of non positivity of $W_{0}$, since $\nu_{0}(y) \sim \sqrt{y}$ as $y \rightarrow+\infty$. An elementary calculation shows that $h_{\delta}$ reaches its maximum (for $y>y_{1}$ ) at

$$
y=y_{M}:=\left(\frac{\sqrt{3} \sqrt{1-\delta}}{\sqrt{y_{1}} \delta}\right)^{2 / 5}>y_{1},
$$

where the inequality comes from (7.1) and from the fact that $\delta<2 / 3$. From (7.5), we obtain

$$
h_{\delta}\left(y_{M}\right) \leqslant \min \left(\left(\frac{9}{32}\right)^{1 / 3} \frac{\sqrt{y_{1}}}{\sqrt{3}}, \frac{1}{2 \sqrt{3 y_{1}}}\right)+\frac{\delta y_{1}^{5 / 2}}{8 \sqrt{3}}-\frac{5 y_{1}^{1 / 10} \delta^{1 / 5}(1-\delta)^{2 / 5}}{8 \cdot 3^{1 / 10}} .
$$

For $\delta=1 / 3$, elementary calculations show that the right hand side in (7.9) is strictly negative for any $y_{1} \in\left(0,(3 / 2)^{1 / 3}\right)$, which implies that $y_{3}(1 / 3)=+\infty$ and completes the proof of the lemma.

\section{Proof of Lemma 2.5}

We denote

$$
U_{\varepsilon}(z)=W_{0}\left(\varepsilon^{-2 / 3}-\varepsilon^{2 / 3}|z|^{2}\right), \quad z \in \mathbb{R}^{d} .
$$

We are going to show that there exists a constant $C>0$ such that for $\varepsilon>0$ sufficiently small, for every ball $B \subset \mathbb{R}^{d}$,

$$
\max _{z \in B} U_{\varepsilon}(z) \leqslant \frac{C}{|B|} \int_{B} U_{\varepsilon}(z) d z
$$

According to Theorem 0.3 in [Sh], Lemma 2.5 follows. First, we notice that, thanks to Lemma 2.2 and (2.15), there exist $C_{1}, C_{2}>0$ such that for every $y \in \mathbb{R}$,

$$
C_{1}(1+|y|) \leqslant W_{0}(y) \leqslant C_{2}(1+|y|) .
$$

Given $z_{0} \in \mathbb{R}^{d}$ and $r>0$, as $z$ describes $B\left(z_{0}, r\right),|z|$ describes the interval $\left[\left|z_{0}\right|-r,\left|z_{0}\right|+r\right]$ if $\left|z_{0}\right| \geqslant r$ and the interval $\left[0,\left|z_{0}\right|+r\right]$ if $\left|z_{0}\right| \leqslant r$. Since the function

$$
f(s)=\left|\varepsilon^{-2 / 3}-\varepsilon^{2 / 3} s^{2}\right|, \quad s \in \mathbb{R}_{+}
$$

is decreasing on $\left[0, \varepsilon^{-2 / 3}\right]$ and increasing on $\left[\varepsilon^{-2 / 3},+\infty\right)$, we infer that $\max \left\{f(|z|), z \in B\left(z_{0}, r\right)\right\}$ can only take the three different values depending on $z_{0}$ and $r$ : either

$$
\left.\max _{z \in B\left(z_{0}, r\right)} f(|z|)=\varepsilon^{2 / 3}\left(\left|z_{0}\right|+r\right)^{2}-\varepsilon^{-2 / 3} \quad \text { and } \quad\left|z_{0}\right|+r \geqslant \varepsilon^{-2 / 3} \quad \text { (case } 1\right),
$$

or

$$
\max _{z \in B\left(z_{0}, r\right)} f(|z|)=\varepsilon^{-2 / 3}-\varepsilon^{2 / 3}\left(\left|z_{0}\right|-r\right)^{2} \quad \text { and } \quad 0 \leqslant\left|z_{0}\right|-r \leqslant \varepsilon^{-2 / 3} \quad \text { (case 2), }
$$

or

$$
\max _{z \in B\left(z_{0}, r\right)} f(|z|)=\varepsilon^{-2 / 3} \text { and } \quad\left|z_{0}\right|-r \leqslant 0 \quad \text { (case 3). }
$$


We are next going to prove (8.1) in each of these 3 cases.

Case 1. We first show that for every $z_{0}, r$ like in case 1 , we have

$$
\left|z_{0}\right|+\frac{r}{\sqrt{2}} \geqslant \varepsilon^{-2 / 3}
$$

Under the extra assumption

$$
\varepsilon^{2 / 3}\left(\left|z_{0}\right|+r\right)^{2}-\varepsilon^{-2 / 3} \geqslant \varepsilon^{-2 / 3},
$$

(8.3) clearly holds. On the other side, if (8.4) is not true, then $\left|z_{0}\right|-r>0$ since otherwise, $0 \in\left[\left|z_{0}\right|-r,\left|z_{0}\right|+r\right]$ and $\max \left\{f(|z|), z \in B\left(z_{0}, r\right)\right\} \geqslant f(0)=\varepsilon^{-2 / 3}$, contradicting the assumption that we are in case 1 . Then, we also have

$$
\varepsilon^{2 / 3}\left(\left|z_{0}\right|+r\right)^{2}-\varepsilon^{-2 / 3}=f\left(\left|z_{0}\right|+r\right) \geqslant f\left(\left|z_{0}\right|-r\right) \geqslant \varepsilon^{-2 / 3}-\varepsilon^{2 / 3}\left(\left|z_{0}\right|-r\right)^{2},
$$

which can be rewritten as

$$
\left|z_{0}\right|^{2}+r^{2} \geqslant \varepsilon^{-4 / 3}
$$

Since $r<\left|z_{0}\right|$, we deduce

$$
\varepsilon^{-4 / 3} \leqslant\left|z_{0}\right|^{2}+r^{2} \leqslant\left|z_{0}\right|^{2}+\frac{r^{2}}{2}+\sqrt{2} r\left|z_{0}\right|=\left(\left|z_{0}\right|+\frac{r}{\sqrt{2}}\right)^{2},
$$

which means that (8.3) also holds if (8.4) is not true. Let $\alpha=\sqrt{3} / 2 \geqslant 1 / 2+\sqrt{2} / 4$. Then,

$$
\begin{aligned}
& \left(\varepsilon^{2 / 3}\left(\left|z_{0}\right|+\alpha r\right)^{2}-\varepsilon^{-2 / 3}\right)-\frac{1}{2}\left(\varepsilon^{2 / 3}\left(\left|z_{0}\right|+r\right)^{2}-\varepsilon^{-2 / 3}\right)-\frac{1}{2}\left(\varepsilon^{2 / 3}\left(\left|z_{0}\right|+\frac{r}{\sqrt{2}}\right)^{2}-\varepsilon^{-2 / 3}\right) \\
& =2 \varepsilon^{2 / 3}\left|z_{0}\right| r\left(\alpha-\frac{1}{2}-\frac{1}{2 \sqrt{2}}\right)+\varepsilon^{2 / 3} r^{2}\left(\alpha^{2}-1 / 2-1 / 4\right) .
\end{aligned}
$$

We deduce from (8.3) and (8.5) that for every $z \in B\left(z_{0}, r\right)$ such that $|z|>\left|z_{0}\right|+\alpha r$,

$$
\begin{aligned}
f(|z|) & =\varepsilon^{2 / 3}|z|^{2}-\varepsilon^{-2 / 3} \geqslant \varepsilon^{2 / 3}\left(\left|z_{0}\right|+\alpha r\right)^{2}-\varepsilon^{-2 / 3} \\
& \geqslant \frac{1}{2}\left(\varepsilon^{2 / 3}\left(\left|z_{0}\right|+r\right)^{2}-\varepsilon^{-2 / 3}\right)=\frac{1}{2} \max _{z \in B\left(z_{0}, r\right)} f(|z|) .
\end{aligned}
$$

Then, we conclude thanks to (8.2) and (8.6) that

$$
\begin{aligned}
\frac{1}{\left|B\left(z_{0}, r\right)\right|} \int_{B\left(z_{0}, r\right)} U_{\varepsilon}(z) d z & \geqslant \frac{C_{1}}{\left|\mathbb{B}^{d}\right| r^{d}} \int_{B\left(z_{0}, r\right)}(1+f(|z|)) d z \\
& \geqslant \frac{C_{1}}{\left|\mathbb{B}^{d}\right| r^{d}} \int_{B\left(z_{0}, r\right) \backslash B\left(0,\left|z_{0}\right|+\alpha r\right)}(1+f(|z|)) d z \\
& \left.\left.\geqslant \frac{C_{1}}{\left|\mathbb{B}^{d}\right| r^{d}} \int_{\left\{z \in B\left(z_{0}, r\right): z \cdot z_{0}\right.} \geqslant\left|z_{0}\right|+\alpha r\right)\right\} \\
& \geqslant \frac{C_{1} v_{\alpha}}{2\left|\mathbb{B}^{d}\right|} \max _{z \in B\left(z_{0}, r\right)}(1+f(|z|)) \\
& \geqslant \frac{C_{1} v_{\alpha}}{2\left|\mathbb{B}^{d}\right| C_{2}} \max _{z \in B\left(z_{0}, r\right)} U_{\varepsilon}(z)
\end{aligned}
$$


where $v_{\alpha}$ denotes the volume of $\left\{z \in B(0,1): z_{1} \geqslant \alpha\right\}$.

Case 2. The assumption that we are in case 2 implies

$$
\varepsilon^{-2 / 3}-\varepsilon^{2 / 3}\left(\left|z_{0}\right|-r\right)^{2}=f\left(\left|z_{0}\right|-r\right) \geqslant f\left(\left|z_{0}\right|+r\right) \geqslant \varepsilon^{2 / 3}\left(\left|z_{0}\right|+r\right)^{2}-\varepsilon^{-2 / 3},
$$

and thus

$$
\left|z_{0}\right|^{2} \leqslant\left|z_{0}\right|^{2}+r^{2} \leqslant \varepsilon^{-4 / 3}
$$

It follows that

$$
\left(\varepsilon^{-2 / 3}-\varepsilon^{2 / 3}\left(\left|z_{0}\right|-r / 2\right)^{2}\right)-\frac{1}{2}\left(\varepsilon^{-2 / 3}-\varepsilon^{2 / 3}\left(\left|z_{0}\right|-r\right)^{2}\right)=\frac{1}{2}\left(\varepsilon^{-2 / 3}-\varepsilon^{2 / 3}\left|z_{0}\right|^{2}\right)+\frac{\varepsilon^{2 / 3} r^{2}}{4} \geqslant 0 .
$$

We deduce that for every $z \in B\left(z_{0}, r\right)$ such that $|z| \leqslant\left|z_{0}\right|-r / 2$,

$$
f(|z|) \geqslant \frac{1}{2} f\left(\left|z_{0}\right|-r\right)
$$

Then, we show that this last estimates holds as soon as $z \in B\left(z_{0}, r\right)$ and $z \cdot z_{0} /\left|z_{0}\right| \leqslant\left|z_{0}\right|-7 r / 8$. Indeed, under this assumption, Pythagoras' theorem ensures that

$$
\begin{aligned}
|z|^{2} & =\left(z \cdot \frac{z_{0}}{\left|z_{0}\right|}\right)^{2}+\left|z-z_{0}\right|^{2}-\left(\left(z-z_{0}\right) \cdot \frac{z_{0}}{\left|z_{0}\right|}\right)^{2} \\
& \leqslant\left(\left|z_{0}\right|-\frac{7 r}{8}\right)^{2}+r^{2}-\left(\frac{7 r}{8}\right)^{2} \\
& =\left(\left|z_{0}\right|-\frac{r}{2}\right)^{2}-\frac{3 r}{4}\left(\left|z_{0}\right|-r\right) \leqslant\left(\left|z_{0}\right|-\frac{r}{2}\right)^{2} .
\end{aligned}
$$

Then, we conclude similarly as in case 1 , thanks to (8.2) and (8.8)

$$
\begin{aligned}
\frac{1}{\left|B\left(z_{0}, r\right)\right|} \int_{B\left(z_{0}, r\right)} U_{\varepsilon}(z) d z & \geqslant \frac{C_{1}}{\left|\mathbb{B}^{d}\right| r^{d}} \int_{B\left(z_{0}, r\right)}(1+f(|z|)) d z \\
& \left.\geqslant \frac{C_{1}}{\left|\mathbb{B}^{d}\right| r^{d}} \int_{\left\{z \in B\left(z_{0}, r\right): z \cdot z_{0} \mid\right.} \leqslant\left|z_{0}\right|-7 r / 8\right\} \\
& \geqslant \frac{C_{1} v_{7 / 8}}{2\left|\mathbb{B}^{d}\right|} \max _{z \in B\left(z_{0}, r\right)}(1+f(|z|)) \\
& \geqslant \frac{C_{1} v_{7 / 8}}{2\left|\mathbb{B}^{d}\right| C_{2}} \max _{z \in B\left(z_{0}, r\right)} U_{\varepsilon}(z) .
\end{aligned}
$$

Case 3. First, we notice that the assumption that we are in case 3 yields

$$
\varepsilon^{-2 / 3} \geqslant f\left(\left|z_{0}\right|+r\right) \geqslant \varepsilon^{2 / 3}\left(\left|z_{0}\right|+r\right)^{2}-\varepsilon^{-2 / 3},
$$

which gives

$$
\left|z_{0}\right|+r<\sqrt{2} \varepsilon^{-2 / 3}
$$

Thus, since $\left|z_{0}\right| \leqslant r$, we get

$$
\left|z_{0}\right| \leqslant \frac{1}{\sqrt{2}} \varepsilon^{-2 / 3} .
$$


If the extra assumption

$$
r \geqslant 5\left|z_{0}\right| / 4
$$

holds, then (8.11) and the triangular inequality give $B(0, r / 5) \subset B\left(z_{0}, r\right)$. Moreover, if $z \in$ $B(0, r / 5)$, then we get from $(8.10)$

$$
\varepsilon^{-2 / 3}-\varepsilon^{2 / 3}|z|^{2} \geqslant 23 \varepsilon^{-2 / 3} / 25 .
$$

Then, we conclude similarly as in cases 1 and 2 :

$$
\begin{aligned}
\frac{1}{\left|B\left(z_{0}, r\right)\right|} \int_{B\left(z_{0}, r\right)} U_{\varepsilon}(z) d z & \geqslant \frac{C_{1}}{\left|\mathbb{B}^{d}\right| r^{d}} \int_{B(0, r / 5)}\left(1+23 \varepsilon^{-2 / 3} / 25\right) d z \\
& \geqslant \frac{23 C_{1}}{25 \cdot 3^{d} C_{2}} \max _{z \in B\left(z_{0}, r\right)} U_{\varepsilon}(z) .
\end{aligned}
$$

As for the last case when (8.12) is not true, we have then

$$
\left\{z \in B\left(z_{0}, r\right): z \cdot \frac{z_{0}}{\left|z_{0}\right|} \leqslant \frac{7 r}{40}\right\} \subset B\left(z_{0}, r\right) \cap B\left(0,\left|z_{0}\right|\right) .
$$

Indeed, using also $\left|z_{0}\right| \leqslant r \leqslant \frac{5\left|z_{0}\right|}{4}$, we have then

$$
\begin{aligned}
|z|^{2} & =\left(z \cdot \frac{z_{0}}{\left|z_{0}\right|}\right)^{2}+\left|z-z_{0}\right|^{2}-\left(\left|z_{0}\right|-z \cdot \frac{z_{0}}{\left|z_{0}\right|}\right)^{2}=\left|z-z_{0}\right|^{2}-\left|z_{0}\right|^{2}+2\left|z_{0}\right| z \cdot \frac{z_{0}}{\left|z_{0}\right|} \\
& \leqslant r^{2}-\left|z_{0}\right|^{2}+\frac{7 r\left|z_{0}\right|}{20} \leqslant\left|z_{0}\right|^{2} .
\end{aligned}
$$

On the other side, for $z \in B\left(0,\left|z_{0}\right|\right)$, thanks to (8.11), we have

$$
f(|z|)=\varepsilon^{-2 / 3}-\varepsilon^{2 / 3}|z|^{2} \geqslant \varepsilon^{-2 / 3} / 2 .
$$

Then, we conclude similarly as in the previous cases:

$$
\begin{aligned}
\frac{1}{\left|B\left(z_{0}, r\right)\right|} \int_{B\left(z_{0}, r\right)} U_{\varepsilon}(z) d z & \geqslant \frac{C_{1}}{\left|\mathbb{B}^{d}\right| r^{d}} \int_{B\left(z_{0}, r\right)}(1+f(|z|)) d z \\
& \left.\geqslant \frac{C_{1}}{\left|\mathbb{B}^{d}\right| r^{d}} \int_{\left\{z \in B\left(z_{0}, r\right), z \cdot z_{0}\right.} \leqslant 7 r / 40\right\} \\
& \geqslant \frac{C_{1} v_{33 / 40}}{2\left|\mathbb{B}^{d}\right| C_{2}} \max _{z \in B\left(z_{0}, r\right)} U_{\varepsilon}(z) .
\end{aligned}
$$

¿From (8.7), (8.9), (8.14) and (8.16), we infer that (8.1) holds, with

$$
C=\min \left(\frac{C_{1} v_{\alpha}}{2\left|\mathbb{B}^{d}\right| C_{2}}, \frac{C_{1} v_{7 / 8}}{2\left|\mathbb{B}^{d}\right| C_{2}}, \frac{23 C_{1}}{25 \cdot 3^{d} C_{2}}, \frac{C_{1} v_{33 / 40}}{2\left|\mathbb{B}^{d}\right| C_{2}}\right),
$$

which completes the Proof of the lemma.

Aknowledgements. Clément Gallo wishes to thank Reika Fukuizumi and Eiji Yanagida for helpful discussions. 


\section{References}

[AAB] A. Aftalion, S. Alama, and L. Bronsard, Giant Vortex and the Breakdown of Strong Pinning in a Rotating Bose-Einstein Condensate, Arch. Rat. Mech. Anal., 178, 247-286 (2005)

[A] S. Agmon, Bounds on exponential decay of eigenfunctions of Schrödinger operators., Lecture Notes in Math., 1159, Springer, Berlin, 1-38 (1985)

[BTnN] S. Boscolo, S.K. Turitsyn, V.Yu. Novokshenov, and J.H. Nijhof, Self-similar parabolic optical solitary waves, Theor. Math. Phys. 133, 1647-1656 (2002)

[BO] H. BrezIS and L. Oswald, Remarks on sublinear elliptic equations, Nonlinear Anal. 10, 55-64 (1986)

[BDS] J. Brüning, S.Yu. Dobrokhotov, and E.S. Semenov, Unstable closed trajectories, librations and splitting of the lowest eigenvalues in quantum double well problem, Regul. Chaotic Dyn. 11, no. 2, 167-180 (2006)

[GP] C. Gallo and D. Pelinovsky, Eigenvalues of a nonlinear ground state in the ThomasFermi approximation, J. Math. Anal. Appl. 355, 495-?526 (2009)

[Fed] M.V. Fedoryuk, Asymptotics of the discrete spectrum of the operator $w^{\prime \prime}(x)-$ $\lambda^{2} p(x) w(x)=0$, Mat. Sb. 68, No. 1, 81-110 (1965)

[Fer] E. Fermi, Statistical method of investigating electrons in atoms, Z. Phys. 48, 73-79 (1928)

[FIKN] A.S. Fokas, A.R. Its, A.A. Kapaev and V.Y. Novokshenov, Painlevé Transcendents, The Riemann-Hilbert Approach, Mathematical Surveys and Monographs, 128. AMS, Providence, RI, 2006.

[GT] D. Gilbarg and N.S. Trudinger, Elliptic Partial Differential Equations of Second Order, Classics in Mathematics, Springer-Verlag, Berlin, 2001.

[GR] I.S. Gradshteyn and I.M. Ryzhik, Table of integrals, series and products, 6th edition, (Academic Press, 2005)

[HM] S.P. Hastings and J.B. McLeod, A boundary Value Problem Associated with the Second Painlevé Transcendent and the Korteweg-de Vries Equation, Arch. Rat. Mec. Anal., 73, 31-51 (1980)

[IM] R. Ignat and V. MiLlot, The critical velocity for vortex existence in a two-dimensional rotating Bose-Einstein condensate, J. Funct. Anal. 233, 260-306 (2006)

[KK] V.V. Konotop and P.G. Kevrekidis, Bohr-Sommerfeld quantization condition for the Gross-Pitaevskii equation, Phys. Rev. Lett. 91, 230402-4 (2003)

[PS] L. Pitaevski and S. Stringari, Bose-Einstein Condensation, (Oxford University Press, Oxford, 2003) 
[Sh] Z. SHEn, $L^{p}$ estimates for Schrödinger operators with certain potentials, Ann. Inst. Fourier 45, 2, 513-546 (1995)

[Su] C.V. Sukumar, Sum rules for confining potentials, arXiv:quant-ph/0611066 (2006)

[T] L.H. Thomas, The calculation of atomic fields, Proc. Cambridge Philos. Soc. 23, 542 (1927)

[ZAKP] D.A. Zezyulin, G.L. Alfimov, V.V. Konotop and V.M. PÉrez-García, Stability of excited states of a Bose-Einstein condensate in an anharmonic trap, Phys. Rev. A 78, 013606 (2008) 